\title{
Author Program Code
}

Aaserud, David TPG 288

Abashkin, V. M. WPF 160

Abbott, Frank TPE 156

Abian, Joaquin TPB 091

Abney, Kent MOF 04:20

Abolin, Craig WOA 05:00, WOB 04:00, WPG 173

Abolin, Craig R. FOC 11:30

Abrams, Garth TPB 099

Abrams, Suzanne TPB 099

Abramson, Fred P. WPG 194

Abushamaa, Amir TPF 224

Acher, Mandelbaum MPA 034

Ackermann, Bradley TPB 071, TPB 051

Ackermans, P. WPF 138

Ackley, Kathryn WPC 073

Adams, Gregory W. TPG 263

Adams, Jeanette TOC 10:10, TPF 246, MPF 250, MPF 249, TPA 003, MPE 230

Adams, Nathan MPB 094

Adams, Nigel G. ThPA 016, MPA 033, WOE 10:30

Addona, Theresa A. ThPG 282

Aduru, Subhodaya ThPE 177

Aebi, Beat WPH 208

Afzaal, Sunia WPA 017

Aggarwal, Suresht K. MPC 130, MPC 129

Agocs, Lisa WPD 093

Agostara, Giuseppina TPE 186

$\mathrm{Ai}$, Jiu MPC 127

Aicher, Klaus-Peter ThPB 055

Akashi, Satoko MPF 257, MPF 256

Akbari, Nisar TPE 181

Aktories, Klaus ThPG 270

Al-Mahmoud, M. S. TPD 146

Alain, Brunot ThPF 222

Alameddin, N. G. WPA 008

Albersheim, P. ThPE 157

Alcaraz, Armando MPD 155

Aldrich, Jane V. MOD 11:50

Aldridge, John TPB 102

Alewood, Paul F. ThPG 278

Alexander, Anthony J. TPB 066, ThPF 239

Alexander, Michael WOA 05:00

Alexander, Michael S. FOC 11:30

Alimpiev, S. S. WPI 288

Aline, Leroy TPA 033

Allan, Katherine MPD 164

Allanson, John WPG 187

Allen, David MPD 152

Allen, Mark TPG 284, TPB 082

Allen, T. A. WPD 083

Allison, John WOD 04:40, ThPA 015, TOC 05:00, ThPF

214, ThPF 213, TPG 280, TPG 271, ThPF 215, WPA 009, ThPF 216

Allman, S. L. WPI 259, ThPF 220, MPF 237
Altman, Elenora I-2 ThOB 11:30

Alvarez, Erwin J. ThPC 119

Amarbalita, Marvy ThPG 276

Ambrus, Gabor MPF 273

Amft, Matthias ThPF 230, WPI 238

Amirav, Aviv TPB 111, MPB 066

Amster, I. Jonathan MPB 083, FOA 10:50, WPE 110, ThPB 060

Amster, Jon WPE 109

Amundson, Dan WPI 255

Amy, J. ThPC 111

An, Yan TPA 019

Anacleto, Joseph F. TPB 082, TPG 284

Anastasio, M. B. MPF 288

Anastassopoulou, J. ThPA 042, TPF 245

Anderegg, Robert J. FOD 11:10

Anders, M. W. MPA 047

Anderson, Gordon ThPB 073, WPE 115, WOC 05:00

Anderson, Gordon A. ThPB 066, MOD 05:000, WPE 114, WPE 113, TOC 04:40

Anderson, J. E. WPD 083

Anderson, J. S. WPE 105, WPE 103

Anderson, Ken B. ThOE 10:30

Anderson, Kerry ThPG 267

Anderson, Mark WPF 132

Anderson, Melissa WPG 174

Anderson, Stephen A. TPA 005

Anderson, Wayne TPE 197

Andri, Tambuti TPA 033

Andren, Per E. MPF 243, TPE 150

Andresen, Brian D. MPD 155, ThPC 120, ThOC 04:20

Andrews, Glenn MPF 281

Andrews, Kim MPA 016

Andrews, Lori E. TPG 255

Andrews, Philip WPI 249

Andrews, Philip C. WPI 246, MPE 203

Andrews, Stuart R. MPA 002

Andrien, Bruce WPH 218

Andrien, Bruce, Jr. MPB 055, ThOD 11:50

Anex, Deon S. MOC 04:00

Angeli, C. WPA 012

Angelico, Vincent TPA 044

Anicich, Vincent MPA 039

Annan, Roland TPG 272, TPG 258

Anren, $\mathrm{Hu}$ WPF 143

Anthony, Lowell TPE 189, TPE 188

Apffel, A. ThPE 151

Aplin, Robin MPE 206, ThPG 267, WPI 294

Appelhans, Anthony WPF 159, ThOF 04:20

Arakawa, Ryuichi MPE 229, TPF 238

Arakawa, Tsutomu ThPG 263

Arata, Yoji MPF 256, MPF 257 
Arimura, Masana ThPD 147

Arisz, Peter W. WPE 125

Arkas, M. ThPA 042

Armentrout, P. B. WPA 001

Armentrout, Peter MOB 04:20

Arnold, Elizabeth WPF 150

Arnold, Randy TOD 04:00, ThPF 229

Arnold, Susan T. WOE 10:50

Arnott, David MOD 10:30, MPF 271

Artaev, Viatcheslav MPB 091, WPF 134, MPD 145

Asada, T. ThPB 079, WPB 039

Asam, Michael ThPE 188

Asano, Keiji ThPC 122

Ashcroft, Alison ThPE 161

Ashcroft, Alison E. TPE 168

Ast, Teodor FOE 11:10

Atal, Ajay WPF 152

Atherton, Paul WPB 043

Aubry, Christiane TPA 021

Audier, Henri ThOA 10:30

Auriola, Seppo I-1 ThOB 11:30

Ausloos, Pierre MPC 100

Avery, Michael TPE 195, TPE 175

Ayer, Stephen W. ThPD 127

Blangerr, Patrick MPB 052

Baba, Aaron I. TPB 079

Babcock, Lucia WPA 019, WPA 020

Babcock, Lucia M. WOE 10:30, ThPA 016

Bach, Gloria A. ThPC 089, ThPC 105

Bach, Stephan B. H. MPD 140, TPD 137

Bachert, Elizabeth TPE 196

Bahr, Ute ThPF 204

Bai, Jian TPF 214, ThPF 210, TPG 251, WPI 260, WPI 241, WPI 262, ThPF 241, FOB 11:30, TPG 292, ThPF 240

Baillie, Thomas A. TOA 09:30

Bajic, Steve TOA 11:10

Baker, Chris MPB 071

Bakhtiar, Ray TOC 10:30

Balazy, Michael ThPD 132

Balcer, Jesse L. MPD 184, MPD 185

Baldwin, Sam TPG 249

Baldwin, Samuel W. TPG 290, TPC 117

Balestra, Michael TPE 161

Balkan, Alexander A. MPB 065

Ball, M. J. MPF 240

Ballard, Kevin TPA 048, TPA 008

Ballinger, William TPE 175

Bamberg, Michael MPB 099

Banda, Phil MPF 247

Banks, J. Fred TPB 083, TPB 086

Banks, Fred ThOD 11:50

Baranov, Vladimir I. TOC 09:30

Barbacci, Elsa MPF 281

Barber, Michael ThOC 04:40

Barbuch, Robert TPB 051

Barcel, Damia TPB 091
Barcelo, D. MPD 172

Barclay, Victoria TPG 282

Bardel, Patrick ThPF 244

Barinaga, Charles J. WPD 079, ThOC 09:30, ThPC 085

Barnidge, D. R. MPF 279

Barofsky, Douglas TPD 125

Barofsky, Douglas F. TPA 040, MOD 11:50

Barofsky, Elisabeth ThPF 208

Barofsky, Lilo TPD 125

Barr, John R. TOB 04:20, MPD 168, ThOF 09:50, TPD 140

Barrelro, Eliezer ThPA 036

Barry, John P. TPF 223

Barshick, C. M. WPD 078, WPD 086

Barshick, Stacy-Ann WPF 131, WPF 130

Barstis, Toni L. O. WPI 286

Barta, Istvan MPF 273

Bartels, Micheal J. WPG 170

Bartlet-Jones, Mike TPG 264

Bartlett, Michael WPD 087, WPD 088

Bartmess, John MPA 008, WPE 111, MPA 016

Barton, Jacqueline K. TPF 233

Bartos, Michael J. ThPA 044

Baschky, Michael TOF 11:10, MOB 10:30

Bashall, Anthony D. TPB 112

Basic, Cecilia ThPC 115

Basile, Franco ThPD 141, ThPC 114

Bastian, Michael WOE 11:10

Bateman, Robert MPB 097

Bateman, Robert H. TPG 263, ThPE 189, MPB 096

Bauer, Jan TOB 11:10, WPF 137, MPC 113

Bauer, M. D. MPF 288, TPA 042, TPG 252

Bauer, Scott WPF 137, MPC 113, MPB 072, TOB 11:10

Bauerle, Gerald MPD 191

Baum, Ellen ThPG 279

Bauschlicher, Charles WPA 014, WPA 013

Bavda, Lalit TPD 133

Bean, Karen MPD 171

Bean, Mark ThOB 04:00, TPG 258

Beasley, Brandon ThPB 072

Beaugrand, C. ThPB 050, WPH 211, ThPB 049, WOB 11:30, ThPA 026

Beavis, Ronald C. TPG 261, ThPF 221, TPG 283

Bebernitz, Geraldine ThPG 279

Beck, Bret WOC 04:00

Beck, Olof TPE 209

Becker, Christopher WPI 264, WPI 263

Becker, Roman MPB 087

Becker, St. WPE 124

Becker, Stefan WOC 04:00

Beckett, Malcolm WPC 066

Beeson, Michelle D. WPH 226, TPB 073, ThPG 256

Begley, Tadgh P. TPG 289

Behme, Robert MPC 136

Behymer, Thomas D. WPF 136, TOB 10:10

Beiersdorfer, Peter WOC 04:00

Belanger, Ronald ThPE 167

Belas, Frank J. TPE 189, TPE 188, TPE 170 
Bclenky, Alexi TPF 217

Bell, David MPF 286

Bencsath, F. Aladar ThPG 264

Bencsura, Akos WPI 244

Benjamin W. WPF 140, WPF 141

Binidicte, Remaud ThPA 027

Benkovic, Stephen J. MPF 258

Bennett, C. B. TPG 252

Bennett, Patrick WPG 168

Bennett, Samuel E. MOD 11:50

Benoit, Frank M. MPD 196, WPF 135, MPD 159

Benson, Linda M. TPD 141, TOA 10:10, FOB 11:10

Bentzley, Catherine TPF 215

Beranova, Sarka TPA 002, ThPA 013

Berg, Fred MPC 116, ThPA 020

Berg, J. M. WPI 237

Bergman, Tomas TPG 268

Bergmann, C. ThPE 157

Bergmann, Frank TPF 235

Berkenkamp, Stefan ThPF 233, ThPG 279, MPC 139, TOD 09:30, MPF 276, MPF 277

Bernard, Escoffier ThPA 027

Bernasek, Steven L. FOE 11:30

Bernier, Ulrich TPE 171

Bernstein, John R. TPE 157

Bertrand, J. Aaron WPD 088

Bertrand, M. ThPA 042

Bertrand, M. J. TPF 245

Bertrand, Michel J. TPA 039, MPC 114, MPB 052, TPF 220, ThPA 041

Bethem, Robert MPD 190

Betowski, L. Don ThPC 121

Betowski, Leon TPA 031, ThPC 123

Betts, Robert ThPA 034

Beussman, Douglas J. MPB 093, TPA 038

Beverly, Michael ThPD 142

Beynon, Robert J. MPF 259

Bezabeh, Dawit WPI 289

Bhavsar, J. H. TPE 166

Bhide, Anjali ThPE 172

$\mathrm{Bi}$, Honggang WOA 11:50

Biemann, Klaus TPG 265, ThPE 149, WPH 217, ThPF 211, MPF 266, ThPE 174

Bier, M. E. TPG 287, TPG 291

Bier, Mark ThPC 095, MPC 120, ThPC 094, ThPC 096, TPG 253, MOD 10:30, MPF 271, ThPC 084

Bier, Mark E. ThOC 11:50, ThPC 098, TPC 117, WPG 178, TPG 290

Bierbaum, Veronica M. TOF 04:40, MPA 009, ThPA 009

Bignozzi, C. Alberto WPD 090

Billedeau, Stanley M. TPB 095

Binder, R. G. TOB 11:30

Bingham, Jon-Paul ThPG 278

Birken, Steven MPF 239

Birkholz, Detlef ThOF 11:50

Bischoff, Rainer TPF 232

Blackburn, R. K. FOB 09:50

Blacken, Craig E. MOF 04:00
Blackledge, James A. ThPF 239, TPB 066

Blackstock, Silas TOF 11:30

Blades, Arthur T. ThOA 09:30, WOB 10:30, TPA 005

Blair, I. A. TPF 244, TPF 229, TPF 225

Blair, Ian A. TPE 170, TPE 189, TPE 188, TPE 200, WPG 199

Blake, Timothy J. TPE 161

Blakley, Calvin ThPF 195

Blanchard, David MPE 233

Blankenship, James ThPA 037, ThPA 038

Bluhm, Brian WPE 118

Blum, Saul ThOE 05:00

Bock, Cheryl TPB 099

Bodor, Nicholas MPF 241

Boger, Dale L. ThPD 126

Bohin, Jean-Pierre ThPE 169

Bohme, Diethard K. TOC 09:30, MPA 032

Boismenu, Daniel TPE 164

Bolanos, B. J. MPB 090

Bolgar, Mark S. TPE 180, MPF 292

Bolton, Barbara A. TPB 106, TPB 105

Bombardt, Paul A. WPG 188

Bond, L. A. MOF 04:40

Bondarenko, Pavel WPH 213

Bonelli, Fabio TPE 186

Bonner, Ron WPB 047, WPB 046, TPG 282, TPG 284

Bookwalter, Craig WPC 070, ThPA 012

Boon, Jaap J. WPC 051, WPC 052, WPE 125, WPE 126

Bopp, H. WPE 123

Borchers, Torsten ThOC 11:50, ThPC 098

Borders, Montie TPG 289

Borgerding, Anthony J. MPD 144

Bori, Shushan TPG 284

Bvmsen, Klaus Olaf ThPE 181

Bortolini, Olga WPD 090

Borum, Peggy TPE 171

Bothner, Brian TPF 241, WPH 207

Bott, Geoffrey ThPE 184, MPB 073

Bott, John D. MPB 073

Boudjouk, Phil WPA 004

Boue, Stephen M. TPA 035

Bourque, Andre TPF 217

Bowers, Larry TPE 211, TPE 212, TOA 05:00, ThPE 155

Bowers, M.T. FOA 09:30, TPB 076

Bowling, Donald J. TPB 096

Box, Harold TPB 077

Boyd, Robert ThPG 273

Boyd, Robert I-1 ThPB 051

Boyer, Scott TPE 192

Boyle, James ThOD 11:50

Braatz, James ThPE 154

Brach, Frank TPD 136

Braddock, W. David FOB 11:10

Bradshaw, Stephen ThPB 078, ThPC 082

Brand, Willi A. WPF 142, WPD 085

Brauer, F. P. MOF 04:40

Braun, Jennifer A. MPA 014 
Braun, Karen P. ThPG 294

Breau, Alan TPE 201, WPG 166

Brenna, J. Thomas WPD 084, MOF 04:00

Brenna, Thomas ThPA 043

Brenneisen, Rudolf TPE 208

Brewer, C. Fred ThPG 249

Briere, Michael A. FOE 11:50

Brigande, John V. ThPE 165

Brimmer, Steven P. ThPE 185

Brink, Christopher MOF 11:10

Brinkmalm, Gunnar TPA 040

Brinkman, U. A. Th. MPD 172

Britt, Phillip F. WPI 261

Brittain, Robert ThPC 123

Brockmann, A. MPF 261

Brodbelt, Jennifer ThOA 12:10, ThPC 117, ThPC 116, ThPC 118, MPA 005, MPD 191

Brodbelt, Jennifer S. ThPC 119

Brodsky, E. S. WPF 139

Brooke, Dana MPC 136

Brooks, James MPD 165

Brown, Bradley TPC 118

Brown, Jeff TPB 110

Brown, Paul W. TPE 181

Brown, Richard MPD 163

Brown, Robert WOD 10:30, ThPF 235

Brown, Stephen M. MPD 185, MPD 184

Brown, Todd WPG 169

Browner, Richard TPB 053

Browning, Martin, Jr. FOC 10:10

Brozovich, Tara WPF 163

Bruce, David WPD 088, WPD 087

Bruce, James WPE 115, WOC 05:00, ThPB 073

Bruce, James E. TPB 077, WPE 114, WPE 113, MOD 05:000, TOC 04:40, TPB 087

Brudny-Kloeppel, Margarete WPG 200

Brumley, William MPD 195, TPA 031

Brumley, William C. TOB 05:00, MPD 202, MPD 201

Brunet, Julie WPF 151

Bryant, Duncan TPB 063

Bryant, Matthew S. TOA 04:20

Brzuzy, L. ThOF 09:30

Buchanan, Michelle WPF 130

Buchanan, Michelle V. TOD 12:10, ThPB 063, WPI 261

Buchbauer, Gerhard MPC 101

Buchsbaum, J. C. WPI 237

Buck, Steven ThPG 289

Buckel, Scott MPF 291

Budde, William L. TOB 10:10

Budzinski, Ed TPB 077

Bukowski, Nick WPG 198

Bunk, David WOA 11:30

Bunn, Alan WPC 066

Burford, Neil WPD 093

Burgoyne, Thomas WPD 080

Burinsky, David MPF 260

Burkhardt, Mark R. TOB 12:10

Burlet-Schiltz, Odile MPF 284
Burley, Stephen K. FOD 12:10

Burlina, A. WPG 201

Burlingame, A. L. ThPG 272

Burlingame, Alma MPF 290

Burlingame, Alma L. TPG 263, TPG 255

Burns, Scot WPD 087

Burrell, Tim MPD 150

Burroughs, John A. FOE 10:30, FOE 12:10

Burton, Lyle WPB 047, WPB 046

Burton, Richard MPA 035

Busch, Kenneth ThPA 032, WPD 088, WPD 087

Busch, Kenneth L. TPA 046, ThPD 139

Buseck, R. Peter MPD 143

Bushnell, J. FOA 09:30

Busman, M. MPF 287, TPG 273

Butala, John MPD 166

Butala, Pranay TPE 193

Butcher, Geoffrey ThPG 281

Butelman, Eduardo MPF 253

Butler, Jeffery M. ThPA 016

Buttrill, S. E., Jr. ThPC 093, MPB 081

Buzy, Armelle ThPG 276, WPC 055

Byrd, Michelle MPA 022

Byun, Jaeman TPF 222

Byun, Yong G. WPA 017, FOA 10:30

Cadiz, Montagu W. MPC 108

Caffrey, Michael ThPG 246

Cai, Jianyi TPE 207, TPB 058

Cai, Zongwei MPD 175

Caimi, Richard J. WPD 084, ThPA 043

Cairns, Stuart N. MPC 128, MPB 054

Callahan, John WPF 132, WPI 266

Callahan, John $\mathrm{H}$. ThPE 170

Callahan, Patrick ThOF 04:40

Camara, Elvira ThPB 064, WOC 11:50

Cameron, Andrew S. WPC 071

Cameron, Douglas WPF 163

Camp II, David G. MPE 213, TPF 234

Campana, Joseph ThOE 04:00, TPA 041

Campbell, Clay TPB 064

Campbell, Dale MPD 170, WPG 168

Campbell, James A. WPF 162, WPF 160

Campbell, V. L. WPE 106, WPE 099, WPE 104

Campo, Karen K. WOD 11:30, ThPF 232, ThPA 039

Campolmi, Laura WPF 156

Canales, Ph.D., Mark FOC 09:50

Canas-Montalvo, Benito WPI 256

Canova-Davis, Eleanor ThPE 148

Cao, Jian-Ru MPB 058

Cao, Ping MPC 126, TPB 084

Cappellini, Luigi WPF 144

Cappiello, Achille MPD 157

Caprioli, Richard M. MOD 04:20, WPI 250, WOA 10:30, WPI 251, MPF 243, TPE 150

Capron, L. WPA 007

Carazzato, Daniel MPB 052 
Carbonell, Ruben G. MPF 251

Carell, Thomas TPC 123

Carlsen, Steve WPG 179

Carlson, Joel MPD 156, WPF 152

Carlson, R. W. ThPE 158

Carlson, Rich TPA 046

Carlson, Shannan TPE 171

Carpenter, Stephen TPD 136

Carr, Scott R. TOC 04:20

Carr, Steven TPG 258, TPG 272, ThOB 04:00, TPC 122

Carrier, Alain MPB 052

Carroll, James ThPB 061, WOC 11:50, ThPE 180

Carson, Peter WPI 284

Cassady, Carolyn J. TOC 11:30, TOC 04:20

Cassidy, Ken C. WPG 172

Castoro, John TPA 041, TPB 078

Castro, Lori MPA 022

Catlow, John TPE 158

Caudill, Doug TPD 14.3

Cavalieri, Ercole TPF 222

Cepa, Steven TPD 133

Cerda, Blas FOA 10:10, TPA 002

Cerniglia, C. E. TPE 155

Cerny, Ronald L. MPB 096, MPD 175

Ch'ang, L. Y. WPI 259

Chace, Donald MPE 209, MPE 221

Chait, Brian T. TPG 281, MPF 238, ThPC 081, ThOC 12:10, WPI 243, TOD 11:50, MPE 210, TPG 283, MPF 239, ThOB 04:40, MPF 255, FOD 12:10, MPF 253

Chakel, J. ThPE 151

Chamberlin, Edwin P. MOF 05:00

Chambers, David M. ThPC 120, ThOC 04:20

Chambers, Hank WPF 128

Chambers, Laura WPF 128

Chan, K. TPE 166

Chan, Kelvin WPH 227

Chan, Stephen MPF 260

Chandrasekher, Charu A. ThPA 018

Chang, Sai ThOC 04:40

Chang, Ted WPC 060

Chang-Ming, Chang WPH 207

Channell, James ThPD 140

Chav, Betty MPF 246

Charbonnier, F. WOB 11:30, WPH 211

Charles, M. Judith TOB 10:30, MPD 151, TPD 127, MPE 216

Chatman, Kelly TPF 241

Chaudhary, A. K. TPF 229, TPF 244, TPE 200, TPF 225

Chaudhary, Ajai K. WPG 199

Chauret, Nathalie TPE 167

Chawla, Rahul TPA 029

Check, Catherine E. MPD 140

Chen, C. H. WPI 259, MPF 237, ThPF 220

Chen, Guodong ThPA 010, TPA 020

Chen, Jim ThOA 12:10

Chen, Ling TPF 242, MPF 247

Chen, Lorenzo H. TPG 289
Chen, Ruidan WPE 114, WPE 113, TOC 04:40, WPE 115, WOC 05:00, MOD 05:000, ThPB 066

Chen, Su ThPE 164, ThPD 129, ThPD 130, ThPE 168

Chen, Tom MOD 04:00

Chen, Xiangjun ThPF 221

Chen, $Y$. ThOB 11:50

Cheng, Michael ThOE 04:40

Cheng, Xueheng TPA 001, TOC 04:40, FOD 10:10, WPH 232, WPE 113, WPE 114, TOC 10:30, MOD 05:000, WPE 115, WOC 05:00

Chernushevich, I. V. FOD 10:30, MPB 085

Chernushevich, Igor MPE 225, ThPG 249

Chevis, Eddie A. ThPD 139

Chiarelli, M. Paul TPG 260

Chien, Benjamin TPB 075

Chilkoti, Ashutosh ThPG 251

Chin-Hsiung, Wang WPH 234

Ching, Jesus ThPF 243

Chinghsiung, Wang WPH 235

Chiu, Chung MPD 161

Chiu, Rick W. TPB 090

Chorush, Russell A. MPA 041

Chott, Robert C. MPE 212, WPH 236

Chou, Chau-Wen WPI 269

Chou, Phillip TOF 11:30, MPA 026

Chow, E. Pingchang MPF 270

Chow, Pauline WPG 196

Chowdhury, Swapan MOD 11:10

Christel, Bourhis MPA 034

Christian, Noah TOD 04:00, ThPF 229

Christopher, Steven J. ThPB 054, WPA 003

Christova-Gueor, Nadia I. TPD 127

Chu, Inhou ThPG 293, ThPG 291

Chu, Yen-Ho FOB 10:10

Chuang, C.H. William WPF 150

Chung, Henry TPB 102

Churchwell, Mona I. TOA 11:10, TPE 206

Chyall, Leonard WOE 11:50, MPA 003

Cisper, M. E. WPF 148

Claereboudt, Jan WPF 138

Claeys, Magda ThPE 168, ThPE 166

Clark, C. R. TPE 206

Clark, Steven D. ThPC 092

Clarke, Nigel J. MPD 153, WPB 042

Clauser, Karl R. TPG 255

Clauss, Scott A. WPF 162

Clauss, Therese W WPF 161

Clay, Keith TOA 04:00

Clemons, P. S. MOF 12:10

Clench, Malcolm MPD 163, MPD 162, MPD 152, WPF 157

Cleven, C. D. ThPC 111

Cleven, Curtis ThPC 109

Clifton, Carol MPC 100

Cockrill, Steve MPF 285

Coddeville, Bernadette ThPE 153

Cody, Robert B. WPB 039, ThPG 294

Cohen, Aharon TPF 217 
Cohen, Gerald ThPE 167

Cohen, Steven L. MPF 238, FOD 12:10

Colby, Steven MPB 086, ThPF 229, ThOD 04:40, ThOF 04:00, TOD 04:00

Cole, Mark TPE 194, TPE 203, TPE 192, TPE 193, TPE 196, TPE 196

Cole, Richard B. TPD 148, WOB 04:20, TPE 162, TPB 081, MPE 223, TPD 134

Coleman, Patricia M. FOC 11:10

Colgan, S. T. FOB 12:10

Collette, Timothy W. WPF 140, WPF 141

Collins, Terrence J. ThPA 044

Colorado, Armando ThPC 117, ThPC 116, ThOA 12:10

Comisarow, Melvin WOC 10:30

Compton, J. L. TPF 229

Compton, Robert ThPA 001

Consler, Thomas MOD 09:50

Cook, Kelsey MPC 103

Cooke, Gary MPC 135

Cooke, Michael WPF 157, MPD 162

Cooks, R. G. MPC 112, ThPC 111

Cooks, R. Graham ThPA 010, FOE 11:10, ThPC 109, MPA 007

Coomber, David C. TPE 161

Coombes, R. C. TPE 153

Cooper, Helen J. TPA 010, ThPA 003

Coopersmith, Brad WOB 04:00

Corbett, J. M. TPG 254

Cordero, M. M. TPG 257

Cordero, Marcela M. MPB 082, TOD 10:30

Corley, Richard A. WPG 170

Cornett, D. Shannon MOC 12:10

Cornett, D. S. ThPF 193

Cornio, L. J. TPB 093

Cornish, Timothy J. MPB 092, MPB 082, TPG 257

Corr, Jay J. WPF 164

Costa, Cesar ThPF 231

Costello, Catherine FOA 11:30, ThPE 163

Costello, Kevin WPI 281

Cotter, R. J. ThPG 280, TPG 257, WPI 237

Cotter, Robert ThOB 11:10, MPF 240

Cotter, Robert J. ThPC 083 TPA 036, MPB 092, MPB 082, TPF 216, TOD 10:30, TPF 216

Cottrell, J. S. TPG 254, WPI 275, TPG 259

Covey, Thomas WOB 11:50

Cox, Kathleen A. TPE 152, MPF 259, TPA 007

Coyle, Robert FOC 09:50

Craig, A. G. ThPE 150

Craig, Anthony G. TPA 010

Craik, Charles MPF 290

Cramer, R. TPF 219

Cramer, Rainer ThPA 040

Cravatt, Benjamin F. ThPD 126

Crea, Attilio TPE 186

Creaser, Colin MPA 025

Creasy, William R. TPB 065

Critchley, Glen MOC 10:10

Crowder, Cathy MPA 016

Crutcher, Richard ThOC 09:50
Cui, Donghui TPE 176

Cummins, Lendell L. ThPG 259

Cunniff, John WPH 219

Curcuruto, Ornella WPD 090

Cureton, G. H. MPC 106

Curtis, Jonathan ThPG 273

Curtis, Jonathan M. ThPD 127, WPD 093

Cusanovitch, Michael ThPG 246

Cusworth, III, William WPH 228

Cyr, Terry TPE 163

Dagan, Shai MPB 066, TPB 111

Dage, Jeffrey TPB 051

D'Agostino, Paul A. MPE 236

Dahl, David WPB 024, WPF 159

Dai, Yuqin ThPF 212

Dalal, Nar S. WPE 120

Dale, Michael WPI 280

Dale, Michael WPI 281, WPI 279

Dale, Mitchell W. WPE 113

Dale, Vanessa MPF 248, ThPG 271

Dalene, Marianne WPG 198

Dalton, A. Leigh WPA 020

Dalton, Howard ThPG 276

Dame, Amy D. MPC 118

Damrauer, Robert TOF 09:30, MPA 038

Danis, Paul O. TOD 04:40

Darsey, Jerry WPB 038

Darst, Seth MPF 255

Darvill, A. ThPE 157

Dasgupta, K. WPA 002

da Silveira, Enio ThPA 034, WPD 089, ThPA 036

Dass, Chhabil TPA 047

Davenport, Michael MPB 077

Davenport, Michael R. MPB 078, MPB 093

Davico, Gustavo E. ThPA 009, MPA 038

Davidson, Susan TPB 062

Davies, Stephen WPI 294

Davis, Dean ThOE 12:10

Davis, Margaret R. TOA 09:30

Davis, Michael T. TPB 067, WPB 026

Davoli, Enrico WPF 144

Dawidski, Jean TPB 077

Dawson, Brian TPE 163

Dayton, David TPA 034

Dear, Gordon TPE 187

Dearden, David MPA 004, ThOA 11:50, MPA 048, TOC 09:50, TOF 12:10

Debbie, Armbrust MPD 181

Dechert, Gary J. TPB 103

Deck, J. TPE 155

Decker, Brian K. WOE 10:30

DeCoster, Donna M. MOC 05:20

de Gouw, Joost A. TOF 04:40

DeGnore, Jon P. ThPC 101

de Hoffmann, E. ThPE 182

de Hoffmann, Edmond ThPD 124

Deinzer, Dr. Max MPD 173 
Deinzer, Max ThOC 05:00, TPD 125

Deinzer, Max L. MPE 227, TPD 142, ThPG 257, MPF 263

Dejsupa, Chadin TOF 12:10, TOC 09:50, MPA 048, ThOA 11:50

De Koster, Chris G. WPE 125, WPC 052, WPE 126

Delacotte, J.-M. WPG 193, WPC 053

Delgado, Nelson MPD 174

Dell, Anne TOD 11:30

Delmore, James WPF 159, ThOF 04:20

DeMaio, William TPE 183

Demirev, Plamen ThPA 035, ThPF 231

den Haan, Joke M. M. ThOB 10:30

Denisov, Eduard V. MPA 050

Denoux, Guy MPD 164

DeNoyer, Lyn TPG 282

DePalma, Patrick, Jr. TPB 094, MOC 04:40

Depperman, Kevin L. MPB 070

DePuy, Charles TOF 09:30, MPA 038

DePuy, Charles H. ThPA 009

DeRoos, Paul C. ThOB 12:10, ThPG 283

Derrick, Peter ThPG 281, MPB 089

Derrick, Peter J. TPA 010, ThPA 003, WPI 256, WPB 033, ThPE 168, ThPG 269

DeRuiter, J. TPE 206

Des Rosiers, Christine WPF 151

DeSantolo, Anthony ThPB 071

DeSantolo, Anthony M. ThPB 067

Desiderio, Dominic MPF 244

Despeyroux, Dominique ThPG 269, MPF 248

Destito, Rosalina TPE 203, TPE 192, TPE 194

Deterding, Leesa WPI 267

Detlev, Suckau ThPF 194

de Vries, Mattanjah S. MOC 04:00

Dharmasiri, Kuruppu ThPG 250

Di Sacco, Sonia MPC 125

Dickey, Robert TPD 135

DiDonato, Gerald WPH 230, TPB 060

Dietrich, G. WPA 002, WPI 290, WPE 122, WPE 124

Dindal, Amy B. WPF 149

Ding, Li N. TOF 04:40

Ding, Wie-Dong ThPG 279

Dingley, David WPB 050, ThPF 234

Diven, Warren TPE 199

Divi, Rao L. ThPG 277

Dixon, Frank, Jr. TPB 050

Dobberstein, Peter WPD 085

Dobson, Christopher WOC 04:40, ThPG 255, MPE 206

Dodd, Jack TPG 282

Dodonov, Alexander F. MPB 065, MPB 079

Dodsworth, David W. TPB 066, ThPF 239

Doerge, D. R. TPE 206

Doerge, Daniel R. ThPG 277, TOA 11:10

Dogruel, David WPI 268, WPI 252

Doktycz, Mitchell J. TOD 12:10

Dollinger, Gavin MOD 10:10

Dolnikowski, Gregory WPB 028

Domon, Bruno ThPE 187
Dong, Xia WPC 054

Dongre, Ashok TPG 266, TPA 015, FOE 10:10

Donnelly, Brian TPE 205

Donnelly, Joseph R. MPD 201, MPD 202, TOB 05:00

Dookeran, Nyla ThPE 176, ThPD 138

Dormagen, Jonas MPB 087

Doroshenko, Vladimir M. ThPC 083, TPA 036, TOD 10:30, ThOB 11:10

Doster, Douglas TPB 097

Douce, David MPD 162

Dougherty, Ralph C. WPF 129

Douglas, Kenneth T. TPE 180

Dourdeville, Theodore TPB 071

Downard, Kevin WPH 217

Drader, J. J. WPE 104, ThPB 074, WPE 099

Dratz, E. A. MPF 279

Dreifuss, Peter ThPE 154, MOC 11:30

Dresch, Thomas ThOD 11:50

Dresch, Tomas MPB 055

Dressler, Rainer WOE 11:10

Drew, Barry TPB 053

Driega, Alex MPD 196

Drinkwater, Donald WPC 068, ThOE 11:10, ThOE 04:20

Driskell, William J. MPD 194

Drucker, Mark B. ThPA 016

Duan, Yixiang WPD 076, MOF 05:00

DuBose, Coit M. ThPD 147

Duchateau, G. S. M. J. E. ThPD 125

Duckworth, Douglas MOF 10:30

Duckworth, Douglas C. MOF 09:50, WPD 077

Duebelbeis, Dennis MPD 185

Duffin, Kevin ThPD 137, WOA 11:10

Duffin, Kevin L. MPB 070, WPH 210

Duhr, Alexander MPB 098

Dull, Gary TPB 102

Dunayevskiy, Yuriy TPC 123, WPF 152, FOB 10:10

Dunbar, Robert C. WPA 023, TOF 11:50, TPA 018, MPA 010

Duncan, Mark W. WOA 04:00

Dunn, M. J. TPG 254

Dunn, Thomas M. WPI 286

Dunphy, Jocelyn C. TOB 04:40

Dunstan, David TPB 099

Dupont, Annick MPE 219

Durkin, David A. FOE 09:30

Dutky, Robert C. MPE 207

Dutton, Walter MOC 11:30

Duursma, Marc C. WPE 125, WPE 126

Dworschak, R. G. ThPF 205, WOD 10:50

Dynin, Efim A. ThPB 056, ThPC 112, ThPC 089

Dyson, $\mathrm{H}$. Jane MPF 258

Eades, Donald TPD 130

Eanes, Ritchie C. WPA 003

Earl, W. L. WPI 291

Easterling, Michael WPE 109, ThPB 060 
Eckart, Klaus ThPG 248, ThPA 022, MPF 289

Eckerskorn, Christoph WPI 257

Edberg, Heather MPB 069

Edinger, Kurt J. MPC 108

Edini, C. WPG 201

Edmonds, Charles G. MOD 11:30, MPF 272

Edmondson, Rick WPI 242

Edmondson, Ricky WOD 04:20, ThPF 236, ThPF 228

Edmondson, Ricky D. ThPF 232, WOD 11:30, ThPA 039

Edwards, Michael WPA 1321

Egge, Heinz ThPE 160

Egsgaard, Helge MPA 031

Ehring, Hanno ThPF 231

Eichinger, Dr. Peter MPD 173

Eichmann, Klaus ThPG 286

Eiden, Gregory C. ThOC 09:30, ThPC 085, WPD 079

Eisenreich, Steven J. ThOF 10:30

Eisman, Mark S. TPE 161

Eitzer, Brian MPD 182

Elicone, Chris TPB 070

Elliott, Steve WOC 04:00

Ellis, Leroy WPC 069

Ellis, P. E. FOA 11:50

Ellison, G. Barney ThPA 009

Emary, W. Bart TPE 181

Emmett, Mark R. MOD 04:20, TPE 150

Eng, Jimmy TPG 279, MPF 294

Eng, Jimmy K. ThPG 283, TPG 278, ThOB 12:10

Eng, Marian ThPE 148

Engelhard, Victor $\mathrm{H}$. ThOB 09:30, ThOB 10:30, ThPG 285

Engelmohr, Peter WPE 096, WPE 095

Engels, J. W. TPF 239, TPF 231

Englehard, V. H. ThOB 11:50

Enke, Christie G. MPB 088, MPB 078, TPA 038, WPD 092, MPB 077, WPB 041, MPB 093

Ens, W. WOD 10:50, ThPF 192, ThPF 205, FOD 10:30, MPB 085

Epstein, Lois B. TPG 255

Erickson, Tina A. TPA 038, MPB 093

Ericsson, Lowell ThPD 128

Eriksson, Jan ThPA 035

Ernest, Marichal ThPF 222

Erve, John TPD 125

Esaw, Barbara TPE 185

Eshraghi, Jamshid MOD 11:10

Ethier, Jean TPE 163

Evans, Jason MPA 029

Evans, Robert MPB 067

Evans, William ]. MOC 05:20

Evard, Dwight WPI 294

Ewing, Andrew TPB 087

Ewing, Nigel TOC 11:30

Eyler, J. R. WPA 008

Eyler, John ThPB 068, TPG 275, TPA 014, MPA 035, MPE 226

Eyler, John R. WPD 078, ThPA 009
F.Barros, Cristina ThPA 033

Fabio C. Gozzo, MPA 007

Fabris, Dan TOD 10:30

Facchetti, S. TPF 239

Fagerquist, Clifton K. TOC 11:50, TOC 04:00

Fainzilber, Michael ThPG 272

Falchetto, Rocco TPG 247, TPB 069

Fales, Henry M. TPA 016, ThPF 205, MPE 207

Falk, Nancy MPC 119

Famiglini, Giorgio MPD 157

Fanelli, Roberto WPF 144

Fannin, Steve ThPE 180, ThPF 199, ThPB 061, WOC 11:50

Farmer, Terry B. WPI 250

Farrell, Nicholas TPA 030

Faubert, Denis MPC 114

Faull, Kym F. ThPE 177

Faye, T. WPG 193

Fearon, Karen TPF 242

Federle, Thomas W. WPF 158

Fedora, Jim W. WPC 072

Fei, Xun ThPF 190

Feinberg, Thomas N. MPD 151, TOB 10:30

Feistner, Gottfried J. WPG 191

Felton, James TPD 130

Fencl, Jennifer B. WPF 155

Feng, Bingbing FOE 11:10

Feng, Rong ThPG 254

Feng, W. Y. ThOA 10:10

Fenn, John B. WOB 12:10, MPB 058

Fenselau, Catherine TPD 145, MPF 295, MPA 006, TOD 10:30

Feny, David TPG 283, MPF 255

Ferguson, James A. ThOE 10:10

Fernandez, Joseph WPI 240

Ferrari, Stefano WPF 144

Ferrell, William WPD 089

Ferrer Correia, A. J. TPB 098, MPA 044, ThPA 033

Ferrige, Anthony G. ThPF 205

Fetterolf, Dean TPE 205

Fields, Steven TPB 071

Fies, Bill ThOC 11:50

Fifer, Robert A. ThPA 030, ThPA 031

Figg, Deborah MOF 11:10

Figliozzi, Gianine M. TPC 117

Filigenzi, Michael MPD 158

Filosa, Angelo MPC 138

Finch, Jeff MPB 059

Finch, Jeffrey W. ThPB 052

Fink, Saul W. MPC 105, MPC 102

Fischer, Steven TPB 052

Fischer, W. H. ThPE 150

Fisher, Dixie TPB 054

Fisher, Keith WPI 295

Fitch, John ThPG 246

Fitch, William TPC 116, WPH 231

Fitzgerald, Michael C. MPE 225

Fitzgerald, Robert WPB 034

Fitzloff, John F. TPB 056 
Fletcher, Michael A. MPD 154

Florance, James MPF 245

Florio, Eugene WPG 180

Flurer, Rick TOA 11:50, TPD 144

Foiles, Linda MPD 192

Foley, B. Lachele ThPA 016

Follansbee, J. C. MPB 084

Foltz, Rodger L. TPE 208

Forch, Brad E. ThPA 031, ThPA 030

Forde, Diane MOD 11:10

Forest, Eric MPF 278, ThPG 260, ThPG 246

Foret, Frantisek MPB 063

Forssmann, Wolf-Georg TPG 248, MPF 254

Foster, Dale R. MPD 188

Fouda, Hassan WPG 167, TPE 194, TPE 195, TPE 192, TPE 213, TPE 193, TPE 175, TPE 203

Fox, Alvin WPF 145

Fox, Karen WPF 145

Frangoice, Fournier ThPA 027, TPA 033, MPA 034

Frank, Samantha MPA 024

Frankevich, V. ThPC 111

Franklin, Anthony M. MPA 037, MPA 018

Franklin, Melanie A. ThPF 199

Franklin, Rebecca TPA 045

Franklin, Ronald B. TPE 157

Franzen, Jochen ThOC 11:10, ThPC 087, ThPC 088

Fraser, Ian WPG 187

Fregien, Kelly TPE 211

Freiser, Ben WPA 013, WPA 014

Freiser, Ben S. WPA 016, FOA 10:30, WPA 015, WPA 017

Freitas, Antonio ThPA 036

Freitas, Michael A. TOA 10:30

Freude, H.-Gerhard TPE 186

Fruchter, John S. WPF 161

Frutos, Dinorah WPD 092

Fuciarelli, Alfred F. TPF 228, MPF 272

Fuentes, Gary R. TPC 114

Fujii, Toshihiro ThPA 021, TPA 025

Fujita, Tsuyoshi ThPF 198

Fujiwara, Hideji MPE 212, WPH 236

Funeriu, Daniel MPE 219

Furlong, Edward T. MPD 160, TOB 12:10

Fusciarelli, Al F. TPF 234

Futrell, J. H. TPA 029

Futrell, Jean H. TPA 028

Gabeler, Stephen TPG 250

Gaelli, Markus ThPB 057

Gage, Douglas A. ThPE 178, WPI 254, TPG 271, TPG 280, ThPG 289

Gagnon, Jean ThPG 260

Gaines, Donald F. MPE 208

Galagan, Natalia MPF 252

Gale, David WPE 115, WOC 05:00

Gale, David C. TPF 234, MPE 213, ThPG 251, TPB 087, MOD 05:000, TOC 04:40

Gallagher, Richard ThPG 281
Gallagher, Richard T. ThPA 003, TPA 010, WPB 033, WPI 256, ThPG 269

Gallagher, Sarah M. ThPA 009

Gallegos, Emilio J. MPB 073

Games, David E. TPE 191

Ganem, Bruce FOD 09:30, TPC 121

Gao, Q. WPH 229, MPB 084

Gao, Quanyin WPH 232, TPA 001

Garbade, Klaus MPA 019

Garcia, Edna WPA 014

Garcia-Romero, Bernado MPD 165

Gard, Eric WOC 11:50, ThPB 064

Gardinali, Piero MPD 177, WPF 128

Gardner, Ben MPD 145, MPB 091, WPF 134

Garner, Carlos O. TPE 170

Garrett, A. W. WPI 291, ThPC 111

Garrett, Aaron ThPC 109

Garrett, Aaron W. ThPC 110

Gaskell, Simon TPA 008, TPA 048

Gaskell, Simon J. TPE 180, TPE 152, MPF 292, MPF 259, TPA 007

Gates, Paul M. TOB 12:10, MPD 160

Gatlin, Christine L. MPE 232, MPE 231

Gaus, Hans ThPG 259

Gauthier, Jeffrey TPE 196, TPE 193, TPE 203, TPE 194

Gedamke, Rich WPH 230

Gellene, Gregory ThPA 017, TOF 05:00

Gellene, Gregory I. ThPA 018

Gelp, Emilioo TPB 091

Gérard, Bolbach ThPF 222

Gerber, Nicholas TPE 178

Gershwin, M. Eric ThPF 242

Geysen, H. Mario TPC 118

Gharahdaghi, Farzin WPI 240

Ghosh, Dipak ThPG 275

Ghosh, Kris MOD 04:00

Gibson, Bradford MPF 242

Gibson, John K. WOD 12:10

Gidden, Jennifer MPA 036

Giese, Roger W. TPF 224

Gilbert, Anthony WPB 043

Gill, C. G. WPI 291, WPD 083, WPF 148

Gillece-Castro, Beth MPF 271

Gillespie, Todd TPE 158

Gillespie, Todd A. WPH 212, TPE 157

Gillig, Kent WPE 118, ThPF 228, WPI 242

Gimon-Kinsel, Mary TPA 006, WPB 030, ThPF 228, ThPF 236

Giordani, Anne B. MPE 224, WPH 220

Giordano, G. WPG 201

Gisclon, Lee G. TPE 160

Glasmachers, Albrecht ThPB 069

Glassmeyer, Susan MPD 198

Gleich, Gerald J. TPD 141

Glish, Gary ThPE 188, TPA 048

Glish, Gary L. MPB 060, MPA 040, WPF 155, TPG 293, ThPC 092, TPA 011, ThPF 197

Glocker, Michael O. FOD 11:50, MPF 263

Gobey, Jason TPB 077 
Gobin, Jovana MPF 242

Godbout, Jerry ThPA 015

Goeringer, Doug E. TPB 088

Goeringer, Douglas ThOC 09:50, MPA 045, ThPC 122

Goeringer, Douglas E. TPA 037, MOF 09:50, TOC $11: 10$

Goetzinger, Wolfgang MPB 063

Goheen, Steve C. WPF 161

Golding, Rafael E. ThPF 209

Goldschmidt, Robert WPI 282, WOD 04:00, ThPF 223, MOC 10:30

Goldthwaite, Charles A. TPE 188, TPE 189, TPE 200

Golovlev, V. V. ThPF 220

Gonnella, Tom TPA 016

Goodall, David M. MPE 205

Gooden, Jonathon TPF 221

Goodley, Paul C. TPB 052

Goodman, Keith J. MOF 04:00

Goodman, Morris ThPG 289

Goodman, Steven R. ThPG 264

Goodner, Kevin L. WPD 078

Goodwin, Mike TPE 158

Goodwin, R. Mike WPH 212

Gordon-Wylie, Scott ThPA 044

Gorshkov, Michael V. WPB 027, ThPB 076

Gvrtler, Bernd ThPB 069

Gottschalk, Russell ThOF 11:50

Goudreau, Paul N. FOD 10:10

Goulmy, Els ThOB 10:30

Graaa, M. TPB 098, ThPA 033

Grabowski, Joseph J. ThPA 008, MPA 014

Grace, Louis I. ThOC 04:20, WPI 286, ThPC 120

Graham, Elizabeth WPG 173

Grande, H. Ray TPD 135

Graner, Helena TPF 224

Grange, Andrew H. MPD 202, TOB 05:00, MPD 201

Grant, Karen E. WPF 160

Grant, R. A. TPG 252

Grant, Russell P. MPA 002, TPE 191

Graves, Peter ThPB 080

Gray, John ThOD 12:10

Grayson, Michael WPC 065

Greaves, John MOC 05:20

Green, Brian ThPE 161

Green, Brian N. ThPG 295, ThPG 252, ThPG 288

Green, John Glass MPC 104

Green, Kirk WOC 11:50

Green, M. Kirk ThPB 064

Green, Martin MPB 097

Green, Martin R. TPG 263

Green, Vaughn E. ThOF 09:50

Greenberg, Gerald E. TPE 198

Greenhill, Paul WPI 295

Gregg, Hugh MPB 069

Greig, Michael J. WOA 10:50, ThPG 259

Griest, Wayne WPF 131

Griffey, Richard H. ThPG 259, WOA 10:50

Griffin, Donald A. TPD 142

Griffin, J. B. WPA 001
Griffin, Patrick TPC 120

Griffin, Timothy ThOC 10:30

Griffith, Kelly S. ThPA 018

Griffiths, William ThPD 144, MPA 011, TPG 268

Grigorean, Gabriela MPB 083

Grigsby, Ronald D. ThOE 11:30

Grimm, Casey TPB 109

Grimm, Deborah A. TPB 079

Grimsrud, A. J. ThPD 145

Grimsrud, E. P. TOF 10:10, MPB 068

Groenewold, Gary WPF 159, ThOF 04:20

Gronert, Scott MOB 04:00, TOA 10:30

Gross, Deborah TOC 04:00

Gross, Deborah S. MPA 043, TOC 11:50

Gross, M. L. WPE 098

Gross, Michael ThPG 255, TPF 221, ThPG 265, TPF 222, ThPG 284

Gross, Michael L. ThPG 287

Grosser, Robert MPD 178

Grossert, S. MPC 112

Grosshans, Peter ThOE 05:00

Grote, William WPC 075

Grotemeyer, Jürgen ThPG 253, ThOA 04:40, ThPA 023, ThPF 230, ThPB 055, WPI 238

Grundwuermer, Johann M. ThPF 230, WPI 238

Gu, Ming MOB 04:40

Guan, Shenheng WPE 116, WPE 119, WPE 120, ThPB 077, ThPF 200, ThPF 202, WPB 040, ThPA 005, WOC 10:50, ThPB 070, WPE 121, ThPF 201, WPF 129, ThPB 065

Guan, Ziqiang TPG 288, MPA 041, MOC 04:20

Guarino, Bradley MPF 281

Guenther, Clemens WPG 200

Guentzel, M. Neal TPD 137

Guerin, Michael WPF 146, WPF 133, WPF 130

Guevremont, Roger WOB 05:00, TPA 004, WPH 225

Guh, H. Y. MPC 137

Guido, David TOA 11:30

Gulcicek, Erol MPB 059

Gulcicek, Erol E. MPB 057

Gulden, Pamela H. ThPG 285, ThOB 11:50

Gunsalus, Irwin ThPG 275

Guo, Lin ThPG 274

Guo, X. Q. WPD 082

Guodong Chen, MPA 007

Gusev, Arkady I. TPE 199, TPE 184, FOB 11:50, WOA 04:20, WIP 274, WOA 04:40

Guy, Philippe ThPG 260

Gwiazda, P. W. FOB 12:10

Haapaniemi, Esa MPC 117

Haas, George W. WPC 069

Haas, Ruth TPE 172

Habibi-Goudarzi, S. TOD 12:10

Habibi-Goudarzi, Sohrab WPH 210

Hachimi, A. WPI 292

Hackett, Murray ThPG 274, TPB 069

Hadad, Christopher MOB 11:10 
Haddix, Martin MPC 103

Haddon, William F. TOB 11:30

Hadfield, Ted L. ThPD 141

Haff, Lawrence TOD 04:20

Haglund, Richard F., Jr. ThPA 040

Haglund, R. F., Jr. TPF 219

Hahner, S. TPF 219

Hahsmi, Mazzaz MPA 047

Hail, Mark TPG 249, MPC 107, WPH 230

Halasinski, Thomas ThPA 015

Halgand, Frdric ThPG 246

Hall, Brad ThPC 118

Hall, Steven C. TPG 255, TPG 263

Hall, Trevor FOC 09:50

Halm, Kathy WPG 186

Hamdan, Mahmoud WPD 090

Hamerly, Thomas ThPB 080

Hamilton, Brenda WPG 173, WOB 04:00

Hamilton, Jill TPF 246

Hamm, H. E. MPF 287

Hammen, P. D. FOB 12:10

Hancock, James R. MPE 236

Hancock, W. S. ThPE 151

Hanisch, Franz-Georg ThPE 161, ThPE 160

Haniu, Mitsuru ThPG 263

Hankin, Joseph MPA 038, TOF 09:30

Hanley, Brian WPI 278

Hanley, Luke FOE 10:30, FOE 12:10

Hansen, E. B., Jr. TPE 206, TPE 155

Hanser, Kent MPB 086, ThOF 04:00

Hanson, Curtiss MPA 027, MPB 094, MPA 024, MPD 150

Hanson, Stewart WPB 043

Hanton, Scott MOC 10:30, WPI 278, ThPF 224, MPB 095, WOD O4:00

Hara, Shinichi ThPG 268

Harden, Charles S. TOF 04:20, ThPA 031, ThPA 030

Harden, Leslie A. TOB 11:30

Harder, Andreas MPA 021

Harder, Scott WPG 182

Harding, Robert H. WPC 067

Haren, Patrick WPH 218, MPB 055, ThOD 11:50

Hargiss, Leonard O. WPC 064

Harms, A. C. WPH 229

Harms, Amy C. TPF 233, FOD 10:10, TPF 234

Harn, Donald A. ThOB 10:10

Harnick, Barry MPD 189

Harnick, Barry J. MPD 188

Harper, C. J. WPE 099

Harris, Frank MPA 011

Harris, Frank M. MPA 002

Harris, Wes WPG 169

Harris, William ThOF 04:00, MPB 086

Harrison, Alex G. WPA 021, WPA 022, TPA 009

Harrison, Evan WPF 159

Harrison, Kathleen WPG 190, ThPD 131

Harrison, M. TPG 259

Hart, Kevin WPB 041

Hart, Kevin J. ThPC 091
Hart, Robert WPG 165

Harvey, David J. ThPE 179, ThPE 189, ThPE 186, ThPE 152

Hass, J. Ronald MPC 131

Hassila, Heikki MPC 117

Hatcher, Joey WPE 111

Hathout, Yetrib MPF 295

Hauer, Charles ThPF 193

Hauer, Michael J. WPG 188

Havel, Jim WPG 184

Hawley-Fedder, Ruth MPD 192

Hayes, Joe WII 266

Hayes, Michael J. WPG 179

Hayes, T. W. WPA 008

Hayes, Timothy WPI 242

Hayward, Mark MPC 110, TPG 266

Hayward, Mark J. FOE 11:30, MPB 075, MPC 111

He, Lin ThPF 190

He, Ling WOD 11:50, TPB 075, TPB 074

He, S. MPF 264

Headley, John V. MPD 183

Hedley, Julie WPC 062, WPC 063

Heeren, Ron M. A. WPE 126, WPE 125

Heikes, David MPD 176

Heimark, L. MPF 262

Heine, Gaby MPF 254

Heinicke, Ralf ThPA 023

Heinze, T. M. TPE 155

Hemberger, P. H. WPI 291, WPF 148, ThPC 111

Hemberger, Philip ThPC 109

Hemberger, Philip H. ThPC 110, WPD 079

Hemling, Mark TPC 122, TPG 258

Hendrickson, C. L. WPE 104, ThPB 074

Hendrickson, Ronald C. ThOB 11:50, TPB 069

Henion, Jack WPG 168, FOC 09:30, WPG 176, WPH 210, TPB 058, MPD 171, WPH 208, MPB 061, WPG 192, MPD 170, MPC 110, TPE 207, WPG 174, WPG 177

Henion, Jack D. FOD 09:30, TPC 121

Henrikson, Steven J. ThPD 126

Henry, Michael S. TPD 134

Hensel, Russell WPI 272

Hentschel, Rosemary T. TPB 096

Hercules, David WPC 054

Hercules, David M. TPE 199, WPI 274, ThOD 10:30,

FOB 11:50, TPE 184, WOA 04:40, WOA 04:20

Herman, Alan ThPG 266

Herold, Christopher WPB 034

Herold, David WPB 034

Herron, Nelson MPD 201

Herron, Nelson R. MPD 145

Herron, William J. TPA 037, TOC 11:10

Hertzberg, Robert TPC 122

Hettich, Robert ThPA 001, WOC 11:30

Hettich, Robert L. ThPB 063

Hewlett, Erik L. ThPG 274

Hieftje, Gary WPD 080

Hieshima, Glenn B. TPB 103

Higgins, Cecil E. WPF 149 
Higgins, Priscilla MPA 008

Hildebrandt, J. D. MPF 287

Hileman, Fred WPC 075, MPC 122, WPG 169

Hileman, Fred D. MPB 070, WPH 210

Hill, Robert TOB 11:50

Hill, Robert H. MPD 194

Hill, Susan E. MPC 136

Hillenkamp, Franz TOD 09:30, ThPF 233, ThPA 040, ThPF 191, ThPF 204, WPI 257, MPF 276, ThPG 279, MPF 277, WPI 247, ThPE 160, ThPE 169, MPC 139, TPF 219

Hiller, Joseph ThPF 215, WOD 04:40

Hiller, Joseph F. ThPF 216

Hilliard, George TPG 258

Hillman, Steven MPE 209, MPE 221

Hilvert, Don MPF 258

Hines, Wade TPA 013

Hipp, Travis L. ThPF 236

Hirabayashi, Atsumu WPH 203

Hirano, Takashi ThPE 171

Hiraoka, Kenzo ThPA 024

Hirayama, Kazuo MPF 256, MPF 257

Hirayama, Shigeki MPA 049

Hirose, Keiji MPA 049

Hirsh, S. MPF 261

Hites, R. A. ThOF 09:30

Hites, Ronald WPD 080, MPD 198

Hkansson, Per ThPA 035

Hlavac, Allan MOD 11:10

Ho, Yen-Peng WPA 023

Ho, Z. Stacy TPB 053

Hkansson, Per TPA 040

Hoekman, Don ThPB 078, WPB 044, WPB 045, ThPB 059

Hoekman, Don J. WPB 042

Hoey, Kenway WPH 207

Hofmann, Bernhard MPB 056

Hofstadler, Steven WOC 05:00, TOC 09:50, ThPB 073, WPE 115

Hofstadler, Steven A. TPB 077, TPB 087, WPE 114, TPA 001, WPE 113, ThPB 066, MOD 05:000, TOC $04: 40$

Hogge, Lawrence TPB 099

Holbert, Anthony TPB 084, MPB 056

Holland, John MPB 091, WPF 134

Holland, John F. MPB 078

Holland, Ricky TPG 260

Holle, Armin ThPF 194, WPI 248

Holliman, Christopher ThPA 002

Holloway, B. ThPE 151

Holmes, Christopher TPC 116

Holmes, John L. MOB 11:30, ThOA 05:00, TPA 019, TPA 020, TPA 021, FOA 12:10

Holthausen, Max C. ThPA 022

Honing, M. MPD 172

Honing, Maarten TPB 091

Hop, Cornelis MOB 04:40

Hop, Cornelis E. C. A. MPE 208

Hop, Marcel, C. E. C. A. ThPA 025
Hopfgartner, Gerard TOA 04:40, TPE 172

Hopkinson, Alan C. MPA 032

Hopkinson, Allen C. TOC 09:30

Hopwood, Femia WPI 295

Horan, G. J. TPA 012, FOB 12:10

Horan, Thomas ThPG 263

Horn, L. ThPC 111

Horn, Lynn ThPA 010

Horvath, Thomas MPB 087

Horwitz, Marcus MPF 242

Hostettmann, Kurt TPE 182

Houalla, Marwan WOA 04:40

Houk, R. S. MOF 12:10

Houston, Chris ThPF 229, TOD 04:00

Houthaeve, Tony TPG 276, FOB 09:30, MPF 283, ThOC 11:30

Howald, Markus MPD 141, MPD 142

Howard, Jack TPB 049

Howard-Lock, Helen E. TPE 154

Howarth, Oliver WPD 091

Howdeshell, Michael MPD 198

Hoyes, John TPG 258

Hsieh, Feng-Yin TPE 200

Hsieh, Frank TPB 070, TPG 250, TPA 013

Hsing-Long, Chei ThPF 225

Hsu, Chang Samuel TPB 103, ThOE 05:00

$\mathrm{Hu}$, Jun ThPA 007, MPA 013

Hu, Peifeng MPF 291, MOD 12:10

Hua, Yousheng TPD 134

Huang, A. Y. ThPG 280

Huang, Chao-Ran TPB 056

Huang, E. MPF 262

Huang, Jianxing ThPD 135

Huang, Lee MPE 214

Huang, Rong ThPG 275, ThPG 290

Huang, S-wan WPH 233

Huang, Yong ThOA 04:00, WPB 030

Huang, Yulin WPE 121, ThPB 070, WPE 119, ThPF 200

Huang, Zhi-Heng ThPE 178

Hubbard, Natalie WPI 247

Huby, Francois TOD 04:40

Huddleston, Michael ThOB 04:00

Hudson, James ThOE 04:40

Huebner, Vreni MOD 10:10

Huggett, Rebert J. TOB 09:30

Hughes, Roger ThOE 12:10

Hull, Clifford ThPE 154, MOC 11:30

Hull, R. Delon MPD 194

Hulse, James WPG 184

Humphrey, Paul WPB 050, ThPF 234

Hunt, Donald F. ThOB 10:30, ThPG 286, ThPG 282, ThPG 285, TPG 264, TPG 247, ThOB 11:50, TPB 069, ThPG 274

Hunt, Jerry E. ThOE 10:30, WPC 069

Hunter, Kevin ThOD 12:10

Hunziker, Heinrich E. MOC 04:00

Hurlbut, Jeff WPG 189 
Hurst, Gregory B. ThPF 219, WPI 261, TOD 12:10, ThPB 063

Husser, Christophe TPE 172, TOA 04:40

Huston, Chuck TPB 107, ThPC 103, ThPC 104

Hutchens, T. William ThPF 242, ThPF 243, ThPF 244

Hutton, Therese ThPG 277

Hwang, Thomas MPA 022

Iannucci, Robert WPG 202

Iden, Charles R. TPF 243, TPF 240, TPF 227

Ii, Tadashi ThPE 159, ThPE 171

Ijames, Carl F. MPE 207, WPE 108

Ikonomou, Michael G. WOB 10:30, WPA 010

Illies, Andreas J. MPA 036, TOF 04:00

Ingedoh, Arnd MPC 139

Ingemann, Steen TPA 027

Ingendoh, A. WPI 275, TPG 259

Ingendoh, Arnd TPG 262, MPF 274, ThPG 296, MPF 275

Ingram, Jani ThOF 04:20, WPF 159

Inoue, Masayuki MPB 064

Inskeep, Phil TPE 175

Isabelle, Daoust-Maleval TPA 033

Isakson, Peter WOA 11:10

Isbell, John MPA 005

Ishihara, M. ThPB 079

Ishikawa, Keiichiro TPF 238

Ismail, Yasser TPF 224

Isobe, Toshiaki MPF 274, TPG 253, ThPG 296, MPF 275, TPG 262

Isola, Mauro MPC 125

Issakova, Olga TPC 113

Itagaki, Yasuhiro ThPF 198

Iwanaga, M. WPD 081

Jackson, George S. ThPB 077

Jackson, Alan K. MPB 070

Jackson, Christian MOC 12:10

Jackson, Edwin K. WOA 04:20

Jackson, George S. ThPB 070, ThPF 200, ThPF 201

Jackson, Kenneth TPG 274

Jackson, Thomas MPD 166, MPD 165

Jackson, Tony WPC 056, WPC 057, MOC 10:10

Jacobs, Peter L. ThPE 175

Jacobson, Denley WPA 006, WPA 1321, TPA 016, WPA 004

Jacobson, Myron K. MPC 118

Jacoby, Cliffton B. TOD 05:00

Jacquy, Dissard TPA 033

Jaffee, E. M. ThPG 280

Jaffee, Elizabeth ThOB 11:10

Jalonen, Jorma MPC 117

James, John WPF 154

James, Mary A. MPA 036

James, Mike ThPC 084, ThOC 11:50, ThPC 098, ThPC 095

Janning, P. MPE 217
Janssen, Hans-Gerd TPB 110

Janzen, Edward, G ThPD 147

Jaquinod, Michel ThPG 246

Jardine, I. TPG 287

Jarman, M. TPD 138, TPE 153

Javahery, Gholamreza TOC 09:30

Jayasimhulu, Koka MPD 178

Jayne, John T. WOE 10:50

Jean-Claude, Blais ThPF 222

Jean-Claude, Tabet ThPA 027, ThPC 107, MPA 034, ThPF 222, TPA 033

Jedrzejewski, Paul TPG 270, MPF 293

Jeffrey, William TPG 264

Jenkins, Roger A. WPF 149

Jenkins, Yonchu TPF 233

Jennings, Keith WPC 057, WPC 056, ThPG 292, ThPG

276, ThPG 271, WPC 055, WPD 091, MPF 248

Jennings, Keith R. ThPG 269

Jennings, Richard WPC 066

Jensen, James ThPA 020

Jensen, Ole N. MOD 11:50, TPG 276

Jentaie, Shiea TPE 204, WPH 235, ThPF 225, WPF 143, WPH 234

Jerina, Donald TPF 236

Jesaitis, A. J. MPF 279

Ji, Qinchung MPB 078

Jiang, Kate MPF 246

Jiang, Xudong FOE 11:10, ThOC 12:10

Jin, Lei FOA 11:50

Jirovetz, Leopold MPC 101

Joklle, Guittard ThPF 222

Joensuu, Pdivi MPC 117

Johnson, Eric ThPC 096

Johnson, Andrew ThPG 292

Johnson, Deborah WPC 075

Johnson, Jennifer WPF 134

Johnson, Jodie TPE 171

Johnson, Jodie V. TPA 032, ThPC 115, ThOE 10:10

Johnson, Kenneth L. TPG 286, TPE 177, TPG 267

Johnson, Robert ThOC 04:40

Johnson, Rubert B. ThPG 289

Johnson, Walter TPC 122

Johnston, David MOD 11:10

Johnston, Murray WPI 284, WPI 287, WPC 070, TPF

215, ThPA 012

Johnston, Paul F. WPF 160

Jones, A. Daniel WPI 289

Jones, A. D. TPD 128

Jones, Alun ThPG 278

Jones, Anita WPI 279

Jones, Anne TPE 187, WPG 187

Jones, Dane R. ThPG 294

Jones, David WOD 05:00

Jones, Jennifer FOE 10:10

Jones, Patrick MPF 290

Jonscher, Karen R. ThOC 04:00

Joode, T. de ThPD 125

Joshi, Jill C. WPG 179

Jourdan, Thomas TPE 205 
Juhasz, Peter TOD 04:20, ThPE 174, ThPF 195

Juneau, Paul L. WPH 220

Jurima-Romet, Malle TPE 163

Just, Craig MPB 094

Just, Ingo ThPG 270

K., Wu MPF 264

Kaesermann, Donald MPF 280

Kage, David MPE 226

Kahr, Michael MOF 04:20, MOF 11:10

Kalinoski, Henry T. WPC 064, MPC 119

Kalkum, Markus MPF 263

Kaltashov, Igor MPA 006

Kamada, Kimiko MPA 049

Kambouris, S. J. TPF 244

Kambouris, Sara J. WPG 199

Kamel, Amin TPE 213, TPE 197

Kan, Steven WPA 015

Kan, Steven Z. FOA 10:30, WPA 016, WPA 017

Kanai, Michiko TPG 253, MPF 274, MPF 275, TPG 262

Kanain, Michiko ThPG 296

Kane, Thomas TPA 044

Kaneko, Takeo MPB 064

Kaplan, David MPD 156

Kaplan, David L. MPF 269

Karas, Michael TOD 09:30, ThPF 233, ThPF 191, ThPE 160, WPI 257, ThPE 169

Kardash, Dawn WPA 004, WPA 1321, WPA 006, TPA 016

Karger, Barry L. FOB 10:10, MPB 063

Karnicky, Joseph F. MPB 081, ThPC 093

Karr, Dane WPF 147, ThPB 059, ThPC 082, WPB 044

Kaswan, R. MPF 261

Kass, Steven TOF 11:10, MOB 10:30, TOF 11:10

Kassel, D. B. FOB 09:50

Kassel, Daniel MOD 09:50

Kasthurikrishna, N. MPC 112

Kato, Koichi MPF 256, MPF 257

Kato, M. ThPB 079

Kato, Shuji TOF 04:40

Katritzky, Alan MPA 035

Katta, Viswanatham ThPG 268, ThPG 263

Katzmann, Jerry A. FOB 11:10

Kaur, Surinder MOD 10:10

Kawabata, Shin-ichirou ThPF 234

Kay, Paul ThPD 142

$\mathrm{Ke}, \mathrm{Fu}$ TPA 004

Keane, A. WPI 275, TPG 259

Kebarle, Paul ThOA 09:30, TPA 005, WOB 10:30

Keck, Helmut MPA 026

Keever, Jeff WPI 271, TPB 072

Keevers, Jeff TPB 055

Kelleher, Neil TPG 288

Kelleher, Neil L. TPG 289

Keller, Douglas TPD 136

Kelley, J. M. MOF 04:40

Kelley, Paul ThPC 099, ThPC 082
Kelly, John FOB 10:30

Kelly, Michele A. TPE 198

Kelly, P. B. WPD 083

Kelly, Peter B. WPI 289

Kemp, Craig MPC 135

Kemp, Terry WPC 055

Kemper, P. FOA 09:30

Kenny, Donald ThOF 04:40

Kenny, James ThPF 196

Kent, Stephen H. B. ThOB 04:40

Kent, Steven B. H. MPE 225

Kenttamaa, Hilkka MOB 11:50, MPA 023, ThPB 072, TOF 11:30, MPA 022, MPA 026

Kenttamaa, Hilkka I. MPA 003

Kenyon, George H. TPG 289

Kcough, T. TPA 042, TPC 252

Keough, Thomas TPC 114

Kerns, Edward MPC 107

Kerns, Edward H. TPB 061, MPC 136, TPE 202, MPC 108, MPC 134, TPC 119

Kerr, Kelly E. MPA 033

Kershner, Karen M. TOB 04:40

Kerwin, James ThPD 128

Keusgen, Michael ThPD 127

Kidd, L. R. TPD 129

Kiddy, R. A. MOF 04:40

Kiesel, Jennifer R. MPB 070

Killinger, William E. MOC 04:20

Kilpatrick, Peter K. MPF 251

Kim, Hee-Yong ThPD 146, ThPD 136

Kim, Ho-Seung MPF 241

Kim, Hyun Sik WPF 129, ThPB 070, ThPF 200

$\mathrm{Kim}$, Jin Young MPC 132

Kim, Ok-Hee ThPG 257

Kim, Sung Ho MPC 132

Kim, Tamen WPH 209, WPH 214

Kim, Yeonhee ThOE 10:30

Kimura, Cliff MOB 04:00

King, Fred MOF 10:10

King, Gina M. ThPG 286

King, Kathy MOD 10:30

King, Richard ThPF 223, ThPF 224, MPB 095, WOD O4:00, WPI 272, WPI 278, MOC 10:30

Kinkead, Scott MOF 04:20

Kinsel, Gary WOD 04:20, ThPF 226

Kinumi, Tomoya TPG 274

Kirby, Daniel P. MPB 062, FOB 10:10, MPB 063

Kirchner, Nicholas J. ThPB 056, ThPC 112, ThPC 089

Kirpekar, F. TPF 219

Kirschner, Gunther ThPE 164

Kishi, Hiroshi TPA 025, ThPA 021

Klassen, John S. ThOA 09:30, TPA 005, WOB 10:30

Klatt, Tracey TPC 120

Kleintop, Brent WPH 227

Klinger, George S. WPF 161

Klohr, Steven E. MPC 108, TPB 061, MPC 134, TPC 119

Kloos, Steve WPA 004

Klopchin, Patrick G. WOA 10:50 
Kluge, H.-J. WPE 122, WPA 002, WPI 290, WPE 124 Klujev, N. A. WPF 139

Klunk, Lewis J. TPE 200

Knapp, D. R. TPG 285, TPG 273

Kneen, Kurt WPA 009

Knighton, W. B. TOF 10:10, MPB 068

Knobeler, Markus ThPB 062

Knochenmuss, Richard MPD 143

Knudsen, Jen MPE 206

Ko, Chan Y. MPC 137

Koch, Wolfram ThPA 022

Kochling, Heinrich ThPF 211

Kocis, Petr TPC 113

Koepke, Andreas K. E. MPF 289

Koerber, S. C. ThPE 150

Koester, Carolyn MPD 192

Koester, Carolyn J. ThOC 05:00

Kofel, Peter MPB 080

Koga, Yoshinori TPF 238

Kohler, Martin ThPE 183

Kohn, Harold TPE 152

Koizumi, Hideaki TPB 080, WPH 203

Kok, G. L. WPF 148

Koleis, Janece C. MPD 160

Kolli, V. S. K. ThPE 158, ThPE 157

Kong, Zhengrong ThPG 290

Konteatis, Zenon TPC 120

Koppenaal, David W. ThPC 085, WPD 079, ThOC 09:30

Korfmacher, Walter A. WPG 175, TOA 04:20, WPG 176

Kornfeld, Rich ThPF 196

Kvrnig, Stefan MPC 101

Korsak, Andy ThPC 084

Koski, Alice ThPB 059

Kvster, Claus WPI 248

Koster, Hubert TPF 216

Kotake, Yashige ThPD 147

Koudrikova, Tatiana TPE 178

Koul, Omanand ThPE 163

Koupai-Abyazani, Mohammed R. TPE 185

Kouzes, Richard T. ThPB 076, WPB 027

Kowalski, E. M. TPF 225

Kowalski, Paul ThPF 193

$\mathrm{Kraft}_{\text {, Andreas }}$ MPB 079

Kragelund, Birthe MPE 206

Krahmer, Mark WPF 145

Krause, Joern MPB 080

Krause, Joern L. ThPF 237

Krebs, Joseph F. MPF 258

Kreek, Mary Jeanne MPF 253

Krier, G. WPI 292

Krishnamurthy, M. TPA 029

Krishnamurthy, Thaiya TPF 230

Kristo, Michael ThOD 05:00

Krmer, Roland MPE 219

Krol, George J. WPG 177

Krone, Jennifer R. ThOB 05:00, WPG 195, WPI 252

Kruppa, Gary WPH 205, WOC 04:40, WPH 206
Kruppa, Gary H. MOC 12:10

Krutchinsky, A. N. MPB 085

Kryak, David D. WPF 136, TOB 10:10

Kürner, Roman ThOC 11:30

Kubis, Alan MOD 04:00

Kubota, E. WPD 081

Kubota, Masayuki TPG 274, ThPE 171

Kuchumov, Askar R. ThPG 288

Kuehl, Douglas MPD 167, TPD 147

Kuell, Chris MPE 235

Kuhnen, Florian WPE 094, WPE 097

Kulkarni, Sandhya MOD 11:50

Kunihiro, Fumio ThPB 052

Kunz, Arthur MPF 276

Kurono, Sadamu ThPE 159

Kuzmich, Sandra TPE 198

Kvalo, Loretta T. WPG 171

Kwok, David WPG 196

L. Amado, Francisco MPA 044, ThPA 033

Labdon, James MOD 04:00

Lacey, M. P. TPG 252, TPA 042

Lacey, Martin P. TPC 114

Lacroix, Monique ThPE 153

Lafargue, P. E. WPI 292

Lafleur, Arthur TPB 049

Lafont, R. TPE 191

Lafontaine, Felix MPC 111

Laiko, Victor MPB 079

Laine, Roger MPE 211

Lam, Zamas TPD 136

Lamaat, Shalaby MPD 181

Lamb, Diane TPD 146

Lammert, Stephen A. ThOC 10:10, ThPC 090

Land, A. P. TPG 291, TPG 287

Land, Adrian TPG 253, MPF 271, MOD 10:30

Land, Adrian P. TPC 117, TPG 290, WPG 178

Land, Mark ThPF 226

Lander, Harry M. MPE 210

Lane, W. S. TPG 287

Langevin, Carolyn N. WPG 175, TOA 04:20

Langford, Marian MPA 011

Langford, Marian L. MPB 0053, MPB 054, MPC 128, MPA 018

Lango, Jozsef MPF 273, TPA 023

Langridge-Smith, Patrick WPI 281, WPI 279, WPI 280

Lapiguera, Amelia P. WPG 175, TOA 04:20

Laramee, James ThOC 05:00, MPD 173

Larsen, Barbara TPF 215, MOC 12:10, TPC 115

Larson, Michael MPD 158

Lasater, M. A. MPA 042

Laskey, Rachel TOA 11:30

LaTorre, Estrella MPD 195

Lattimer, Robert WPC 059, WPC 058

Laude, D. A. WPE 107, WPE 105, WPE 102, WPE 106, WPE 100, ThPC 108, MPA 042, WPE 101, ThPB 074, MPB 090, WPE 099, WPE 103, WPE 104

Laukien, Frank ThPB 062, WPH 205, MOC 12:10 
Laurence, Rozes ThPF 222

Lausecker, Berthold TPE 172, TOA 04:40

Lausevic, Mila ThOC 12:10, ThOC 05:00

Lautz, Carsten ThPF 204

Lavine, Gary ThPF 213, ThPF 216, WOD 04:40

Laviolette, Barbara TPE 185

Lawrence, David L. WPF 158

Lay, Jack TPD 132, TPD 131

Lay, Jackson WPB 038, TPG 260

Lay, Jackson O. MPB 074

Le Blanc, J. C. Yves WPH 225

Le, Han TPF 236, TPF 218, ThPG 262, ThPG 261

Le, John C. ThPG 263

Leal, Joco Paulo WPA 011,WPI 293

Leano, Hector WPF 154

Leary, Julie A. ThPE 183, ThPE 185, ThPE 184

Leathard, David MPD 163

I ebedev, A. T. WPF 139

Lebedev, Albert MPC 131

LeBel, Guy L. WPF 135

LeBlanc, Daren J. TPE 154

LeBlanc, Yves MPE 221

Lebrilla, Carlito WOC 11:50, ThPB 061, ThPE 180

Lebrilla, Carlito B. ThPB 064

Lebtrilla, Carilto ThPF 199

Lecchi, Paolo TPF 218, TPF 236, ThPG 262, ThPG 261

Leclercq, Piet A. TPB 110

Lee, Hack Sung MPA 009

Lee, Heewon WPI 253

Lee, K. L. MPF 237

Lee, Michael TPB 060

Lee, Mike MPC 107, WPH 230, TPG 249

Lee, Mike S. TPB 061, TPE 202, MPC 108, MPC 136, MPC 134, TPC 119

Lee, S. TPB 076

Lee, Sam WPA 013

Lee, Sam A. WPA 016, FOA 10:30

Lee, Samuel WPA 015

Lee, Samuel A. WPA 017

Lee, Terry D. TPB 067, ThPF 218, ThPF 217, WPB 026

Lee, Yin-Yu MPA 012

Leeck, Diane ThPB 072, MPA 022

Leeck, Diane T. MPA 003

Lehman-McKeeman, Lois D. TPD 143

Lehmann, Wolf MPF 293

Lehmann, Wolf D. TPG 270

Lehn, Jean-Marie MPE 220, MPE 219

Lei, Qing Paula FOA 10:50, ThPB 060

Leibman, Chris P. MPD 154

Leize, Emmanuelle MPE 220, MPE 219

Lemire, Sharon ThPA 032

Lemoine, Jirtme ThPE 187

Lennon, III, John D. ThPF 197

Lennon, John WOD 10:30

Leonardi, Alfredo WPF 144

Leone, Anna WPF 144

Leone, Stephen R. TOF 04:40

Leone, Stephen R. MPA 012

LePage, Guy TPE 179
Lipine, Frangois MPA 015

Lepine, Francois L. TPE 165

Lerner, Jeremy WPB 034

Lerner, Richard A. ThPD 126

Leroi, George E. WPA 009, ThPA 015

Levendis, Yiannis WPF 152

Levery, Steven B. ThPE 162

Levis, Robert J. WPI 265

Levsen, Karsten TPB 091

Lewer, Paul MPD 184

Lewis, J. Kathleen WPG 195, WPI 252

Lewis, Richard J. ThPG 278

Lewis, Tanya M. MPD 154

Leymarie, N. WPA 012

Li, Chun ThPD 134

Li, Guo-Zhong WPH 209, WPE 120, WPH 214, WPE 121

Li, Ka Wan ThPD 129

Li, Kai-Ming TPF 222

Li, Liang ThPF 212, MOC 11:50, WPH 204, WPI 277, ThPF 209, WPI 245

Li, Lily Y.-T. WPG 192, WPG 177, WPG 168, MPD 170

Li, Nan ThPF 226

Li, Qimin ThPE 166

Li, Rebecca WPB 044, WPB 045, ThPB 078

Li, Ruomei MPA 003

Li, Xia-Ling ThPG 253

Li, Xuhui TPB 089, WOD 11:50

Li, Yaming MPB 064

Li, Yiao-Ling WPI 238

Li, Yunzhi WOC 11:10, WPI 276, TOD 10:10

Liang, Li TPB 075, WOD 11:50, TPB 074

Liang, Xiaoli WPI 241, ThPF 210, ThPF 240, WPI 262, ThPF 241, WPI 260, TPG 251, TPF 214

Liang, Yongjiang MPA 048

Liang, Zhenmin ThOE 05:00

Liao, Pao-Chi ThPF 214, TPG 280, TPG 271, WOD 04:40

Lias, Sharon MPC 100

Lidgard, Ray WOA 04:00

Lifshitz, C. ThOA 10:10

Lim, H.K. TPE 166, WPH 227

Limbek, Bela MPB 081

Limero, Thomas WPF 154

Lin, C. C. WIG 176

Lin, Chin-Chung WPG 175, TOA 04:20

Lin, Chuan-Yuan TPA 018

Lin, Peiping TPB 068

Lin, Tong TPG 293

Lin, Whei ThPA 036

Lin, Zhen TPE 209

Linberg, Leonid WPG 202

Linden, H. Bernhard MPB 099

Linder, Christopher WPA 020, WPA 019

Lindgren, Jan Eke ThPD 144

Lindh, Ingemar TPG 268

Lindinger, M. WPE 122, WPA 002, WPE 124

Lindley, Chris E. TOB 12:10 
Lindley, Patricia M. ThOD 10:10

Lindsay, Tom J. WPH 212

Lindsey, Jean MPB 069

Ling, Victor ThPE 148

Ling, Yong-Chien MOF 11:30

Link, Andrew MPF 294

Linnert, Harrald V. MPA 017

Linscheid, M. MPE 218, MPE 217

Lipsky, James J. TPE 177

Liston, Theodore TPE 196, TPE 203, TPE 192, TPE 194

Little, Daniel P. MOD 04:40, TPG 289, MOC 04:20

Little, James L. WPB 037

Liu, Yan-Hui WPI 262, ThPF 241, TPF 214

Liu, Chuanliang ThPE 155

Liu, Dai-Fang MPF 277

Liu, Fei MPB 077

Liu, Naili ThPG 268

Liu, Yan-Hui WPI 260, ThPF 210, WPI 241, TPG 251, ThPF 240

Liu, Yuanhui FOB 11:30

Liu, Zaiyou ThOF 09:50

Lloyd, Patrick MPD 146

Lloyd, Steven TPB 109

Lloyd, Thomas WPG 186

Lobstein, N. WPI 292

Lock, Colin J. L. TPE 154

Loewen, Mark MPD 197

Loftus, Neil WPG 198

Lombardo, Christian MOD 09:50

Lombardozzi, Antonietta MPD 157

Londry, F. A. ThPC 106

Londry, Frank A. ThOC 05:00

Long, Austin WPG 189

Long, David T. ThOF 10:30

Long, S. Randolph TPF 230

Lonning, P. E. TPD 138

Loo, Joseph A. MOD 12:10, MPE 204, WPI 258, MPF 291

Look, Gary TPC 116

Lopata, Ronald J. MPC 111

Loran, John S. MPE 205

Lorenz, Peter TPG 270

Louris, John ThPC 099, ThPC 082

Lowen, M. ThOF 11:10

Lowes, Stephen TPE 168

Lowes, Steve WPF 138

Lu, Hsieng ThPG 268

Lu, Lin WPB 043

Lu, Taiwei WPB 034

Lu, Wenzhe TPE 162, TPB 081, MPE 223, TPD 134

Lu, Zhi-Zhen TPB 056

Lubman, David WPI 253, TPB 089, WOD 11:50

Lubman, David M. ThPF 241, WPI 241, ThPF 210, WPI 262, TPF 214, ThPF 240, TPG 251, TPB 074, WPI 286, TPG 292, WPI 260

Lucke, Richard B WPF 161, WPF 162

Ludicky, Robert WPE 127, TPB 097

Luman, David TPB 075

Luman, David M. FOB 11:30
Lund, Anders L. MPF 265

Lundberg, David WOA 11:50

Luo, Wendy TPF 201, WPG 166

Luther, E. W. TPA 012

Luther, El TPE 213

Luther, Elbridge TPE 192, TPE 194, TPE 193, TPE 203

Luther, Michael MOD 09:50

Lützenkirchen, K. WPI 290, WPE 123, WPE 122, WPA 002, WPE 124

Lykins, Jr. WPF 140, WPF 141

Lyons, L. E. FOA 11:50

M. Domingues, M. Rosrio TPB 098

Ma, Cheng-Yu WPF 149

$\mathrm{Ma}, \mathrm{Li}$ TPD 140, MPD 168

Ma, Nina TPC 113

Ma, Yee-Chung ThPD 136, ThPD 146

Maass, Imke ThPB 069

MacDonald, Linda M. MPD 187

Macfarlane, Ronald WPH 213

Macfarlane, Ronald D. MPF 285

Mackintosh, J. Gordon WPI 280

MacLean, Matthew TPB 112

MacMillan, Denise TOA 04:00

Maden, Amy J. MPB 075

Magarotto, M. WPG 201

Maggio, Vincent L. MPD 168, TOB 04:20

Magiera, Daniel TPF 224

Mahon, Andrea WPC 055

Maier, Claudia S. ThPG 257

Maisey, Ann MOC 10:10

Maisey, Anne TPG 263

Maisey, E. Anne ThPE 186

Maitre, P. FOA 09:30

Majidi, Vahid WPC 073, ThPA 004

Major, Hilary J. TPE 168

Makarov, Alexander MPB 089, ThPB 053

Mdkeld, Mauri MPC 117

Makino, S. WPB 039

Malaiyandi, Murugan MPD 196

Malek, Robert ThPB 075

Maleknia, Simin D. ThPF 245

Malfatti, Michael TPD 130

Malis, Greg M. WPG 185

Mallis, Larry M. TPB 059, MPC 105

Malspeis, Louis WPG 197, PC 123

Mamer, Orval A. TPE 165, MPA 015, WPF 151

Mammen, Mathai WPH 232

Managadze, George WOE 12:10

Mandich, Mary ThPB 071

Mandich, Mary L. ThPB 067

Mann, M. TPF 231

Mann, Matthias FOB 09:30, TPG 277, TPG 276, MOD 09:30, ThOC 11:30, ThPF 204, MPF 283, WPB 046, WPH 206, ThPG 270

Mansoori, Bashir WPI 287

Manuelli, P. WPI 292

Manura, John MPB 071, MPC 109 
Manzella, Lynn M. FOE 11:30

Margalo, Joaquim WPA 011, WPI 293

March, R. E. ThPC 106

March, Raymond E. MPA 032, MPD 199, ThOC 12:10, ThOF 11:30, ThOC 05:00

Marchese, Alfredo WOA 11:50

Marcos Eberlin, MPA 007

Marcotte, I. TPF 245, ThPA 042

Marcus, Alvin ThPC 123, MPD 195

Marcus, R. Kenneth MOF 11:50, MOC 04:40, WPA 003, ThPB 054, TPB 094

Margalit, Alon WOA 11:10

Marie-Claude, Perlat MPA 034

Marion, Didier ThPG 260

Mark, J. FOB 09:50

Markey, S. P. TPB 101, TPB 093

Markey, Sanford P. MPE 207

Markham, Dan A. WPG 170

Marlhne, Pirez-Lumbroso ThPA 027

Marnett, L. J. TPF 229

Marques, O. S. TPB 098, ThPA 033

Marquez, Cristina D. TPE 190

Marquis-Rigault, Annie MPE 219

Marrero, Carlos MPC 111

Marsh, Eugene ThOD 11:30

Marshall, Alan G. WOC 10:50, WPE 116, WPA 018, MPA 046, WPE 120, WPE 119, WPE 121, ThPD 133, ThPB 077, ThPA 005, WPB 040, WPF 129, ThPB 065, ThPB 070, ThPF 200, ThPF 201, ThPF 202

Martin, Charles MPB 083

Martin, Gilbert MPF 268

Martin, Millie WPF 154

Martin, Stephen TOD 04:20, ThPF 195, ThOD 11:10, TPG 250, TPB 070

Martin, Steve TPA 013

Martine, Tessier ThPF 222

Marto, Jarrod A. ThPD 133, ThPB 065, ThPF 202

Masoud, Hussein I-2 ThOB 11:30

Massari, Alessandro MPD 157

Masselon, C. WPI 292

Masson, Jean-Michel MPF 284

Matassa, Luca C. WPG 172

Mathews, Kimberly MPB 056

Mathews, W. Rodney TOA 11:30

Mathur, Chandra WPG 171

Matimba, Henri E. K. TPA 027

Matsui, Neil TPG 255

Matsumoto, Hiroyuki TPG 274

Matsumoto, Richard WPG 166

Matsuo, Takekiyo MPE 229

Matteson, K. J. WPI 259

Mattina, Mary Jane MPD 182

Maunit, B. WPI 292

Mayeno, Arthur N. TPD 141

Mayer, Istvan ThPA 028

Mayer, John ThPG 268

Mayol, Robert F. TPE 200

Mays, Dennis C. TPE 177

Mazurkiewicz, Paul MPD 173
McCandless, Jacqueline MPF 247

McCarthy, Dennis J. TPE 161

McCloskey, James A. TPF 237

McCluer, Robert ThPE 163

McClure, P. Cheryl MPD 168, TPD 140, TOB 04:20

McCormack, Ashley L. ThPG 283, ThOB 12:10

McDonald, Thomas MPD 164

McDowell, Roy A. TOD 11:30

McElvany, Stephen ThPA 002, WPF 132

McEwen, Charles MOC 12:10, TPC 115

McGiff, John C. ThPD 132

McGilvery, D. C. WPB 025

McGown, Steven MPD 174

McGuire, Lisa MOD 10:10

McHugh, Charles WOA 11:50

McHugh, Sean ThPB 078, WPB 045, WPB 044

McIntire, W. E. MPF 287

McIver, Robert, Jr. WOC 11:10

McIver, Robert T., Jr. TOD 10:10, WPI 276

McKay, Richard TPC 115

Mckee, Michael TOF 04:00

McKenna, Rosemary TOA 11:30

McKown, Linda A. TPE 160

McLafferty, Fred TPG 288

McLafferty, Fred W. MOD 04:40, MPA 041, WPE 119, MOC 04:20, TPG 289

McLean, Matthew TPF 242, MPF 247

McLuckey, Scott MPA 045, ThOC 09:50, ThPC 122

McLuckey, Scott A. MOF 09:50, TPA 037, TPB 088, TOD 12:10, TOC 11:10

McMahon, John ThPA 043

McMahon, Terry MOB 10:10, MPA 051, ThOA 04:20, ThOA 10:30

McMarthy, Tom WPI 294

McNett, Debra A. MPD 188

McSpadden, William M. WPB 037

McVeety, Bruce D. WPF 161

McWilliams, Ted B. ThOE 11:30

Medzihradszky, Katalin F. TPG 263

Meffert, Anja ThOA 04:40

Meier, Mark ThPA 004

Meisels, Gerry TPA 026

Mello, Charlene M. MPF 269

Mellon, Fred A. ThPD 130, ThPE 168

Meltzer, Michael MPB 069

Menacherry, Stanley TPD 133

Meng, Shi-Yuan ThPG 268

Mentzer, Mary TPC 122

Menzies, Allan TPE 163

Mercer, Roger S. ThOF 11:30, MPD 199

Merriweather, Roosevelt ThPC 090, WPF 133

Mertens, Thomas R. TOB 04:40

Mesmer, Mantai TPB 092

Mestdagh, H. WPA 007, WPA 012

Metcalfe, Christopher D. ThOC 12:10

Meyer, Jacques MPF 278

Meyer, Timothy R. MPD 187

Michael, Wadsley MPD 181

Michel, Hanspeter TPF 235 
Micke, Mel ThPF 216

Migdalof, Bruce H. WPG 199

Mikaya, Anzor MPC 100

Miles, Walter MPD 161

Milgram, Eric ThPB 068

Miller, Cathy M. TPC 114

Miller, David ThOF 04:20

Miller, Jeffery ThOE 10:30

Miller, John WPI 283

Miller, Mark L. ThPC 113

Miller, Scott FOE 11:10

Milliet, Ariel ThOA 10:30

Millington, David MPE 209

Milot, Sylvain MPA 015

Miranker, Andrew WOC 04:40

Mireault, Pascal MPC 114

Mirza, Urooj A. MPE 210, MPF 239, FOD 12:10

Mische, Sheenah, M. WPI 240

Miskimmin, B. M. ThOF 11:10

Mitchell, Charles WPI 249

Mitchell, Dale W. WPE 117, WPE 112

Mitrovich, Samuel WOB 04:40

Miyares, Paul H. MPB 088

Mlinsky, V. V. WPI 288

Mo, Wenjun MPA 018, MPA 037

Moggi, Marco WPF 144

Mohn, C. ThPE 157

Mohr, Martin ThPE 181

Moini, Mehdi TPB 084, MPC 126, MPB 056

Molinski, Tadeusz F. ThPF 199

Mong, Gary M. WPF 162

Moniatte, Marc MPF 268

Monnig, Curtis A. TPB 090, MPE 205

Montanarella, L. TPF 239

Monteagudo, Edith TPE 186

Monteiro, Candida MPE 228

Monteiro, Carlos A. ThPG 264

Montgomery, Jane TPE 164, WPF 151

Moon, Hong-sik TPE 152

Moore, Christopher MPF 242

Moore, Colin MPD 182

Moore, Robert B. WPH 214, WPH 209

Moore, Theresa A. WPG 188

Mordehai, Alex MPB 081

Morgan, E. D. TPE 191

Morgon, Nelson H. MPA 017

Mori, Brian WPG 196

Morin, Paul E. FOD 10:10

Moritz, Fritz ThOA 04:40, ThPA 023

Morrall, Stephen W. WPF 158

Morrical, Bradley MPD 149

Morris, A. Kirk TPF 234

Morris, F. X. MPC 106

Morris, Howard R. TOD 11:30

Morris, III, Samuel J. TOB 04:40

Morris, Jackie MPD 152

Morris, Michael ThPG 273

Morris, Mike ThPG 295

Morris, Robert A. WOE 10:50
Morrison, R. J. S. WPB 025

Mortensen, Peter ThPF 204, TPG 276

Morton, George TPE 183

Morton, Shelby MOF 10:30

Morton, Thomas Hellman MOB 12:10

Mosbaugh, Dale W. MOD 11:50

Moseiey, Arthur WPG 165, TPE 151

Mosely, Jackie A TPA 010

Moshkarina, N. A. WPF 139

Moss, M. Lee WPG 186

Mouget, Yves TPA 039

Moulis, Jean-Marc MPF 278

Mount, Andrew WPI 280

Mowry, Curtis ThPC 114

Moyer, James MPF 281

Moyer, Michael D. TPE 159

Muddiman, David C. TPE 199, ThOD 10:30, TPE 184

Mueller, Matthias ThPB 055

Muenster, H. MPE 218, MPE 217

Muething, Johannes ThPE 160, ThPE 161

Muir, D. C. G. ThOF 11:10

Muir, Derek MPD 197, MPD 193

Muir, Tom ThOB 04:40

Mulder, Patrick TPF 222

Muller, J. F. WPI 292

Muller, Odillo MPB 063

Mullis, James TPB 054

Mulvana, Dan WPG 168

Munch, Jean W. TOB 10:10

Munchausen, Linda TPB 109

Munson, Burnaby MPA 029, MPA 028

Murad, Edmond WOE 11:10

Murphy, A. Tony WPH 212

Murphy, Anthony T. TPE 157

Murphy, Robert C. WPG 190, TOA 12:10, ThPD 131, TPE 173

Murray, Kermit K. ThPF 190

Murray, Robert TPE 161

Murrell, Jason ThPC 086

Murthy, Satya S. WPG 171

Murugaiah, Veeravagu TPF 224

Musselman, B. ThPB 079, WPD 081

Musselman, Brian D. ThPB 052, MPB 059

Musser, Steven M. FOC 10:10, TPD 135

Myers, James D. WPB 032

Myers, Tanya MPD 198

Mylari, Larry TPE 175

Mylchreest, I. C. TPG 291

Mylchreest, Iain TPB 064, MPC 133

N. Domingues, Pedro ThPA 033, MPA 044

Nadeau, Ronn WPH 236

Nadeau, Ronn G. MPE 212

Naemura, Koichiro MPA 049

Nagabhushan, T. L. MPF 262

Nagai, Yoshitaka ThPE 159

Nagel, Marcus ThPC 088

Naim, Abdul TPF 224 
Nair, Hari TPA 044, FOE 10:10

Naito, Yasuhiro TPF 238

Nakajima, Terumi ThPF 198

Nakamura, Takemichi ThPG 272

Nakata, Hisao ThPA 024

Nakayama, Hiroshi TPG 253, MPF 274, ThPG 296

Nanayakkara, Vajira MPA 026

Naoki, Hideo ThPF 198

Nappi, M. ThPC 111

Narayanaswami, Gowri WPI 265

Nardo, Cymbelene L. TOA 04:20

Nash, John MOB 05:00

Natale, David MOD 04:00

Nauman, David TPB 049

Naven, Thomas J. P. ThPE 179, ThPE 152

Navetta, Kim TPE 213

Nawrocki, Joe TPA 014

Nawrocki, Joseph TPG 275

Naylor, Stephen TOA 10:10, TPD 141, TPG 267, TPG 286, TPE 177

Naylor, Stephen FOB 11:10

Nedved, Michael L. TPC 121

Needham, Larry L. TOB 04:20

Needham, Shane TPE 195

Neidermann, Gabi ThPG 286

Neitz, Susanne MPF 254

Nelson, Chad C. TPE 208

Nelson, Christopher A. ThPG 287

Nelson, Randall W. WPG 195, WPI 252, WPI 268, ThOB 05:00, WPI 269

Nemirovskiy, Olga ThPG 265

Nero, Vince WPC 068, ThOE 11:10

Nero, Vincent ThOE 04:20

Newman, Carolyn M. TOB 04:20

Newman, Michael WPG 192

Newton, Russell P. ThPG 269

$\mathrm{Ng}$, Ken MPE 235

Ng-Ilag, Jocelyn H. ThPG 247

Ngoka, Lambert FOA 11:30

Nguyen, Viet ThPA 025

$\mathrm{Ni}$, Jinsong WPA 022, WPA 021

Ni, Zhi-Jie TPC 116

Nibbering, Nico M. M. TPA 027

Nichol, Greg MPD 201

Nicol, Gordon MPA 029

Nicola, Anthony J. ThOD 10:30, WOA 04:20

Nicoll-Griffith, Deborah TPE 167

Nigam, Somesh MOD 04:00

Nigel Adams WOE 10:30

Nikiforov, Alex MPC 101

Nikiforov, S. M. WPI 288

Nikolaev, Eugene ThOA 04:20, MPA 051

Nikolaev, Eugene N. MPA 050

Niwa, Yoshio MPB 064

Nobes, Brian J. TPE 200

Noble, Christopher MPD 148

Noble, Dick MPF 247

Nock, Steffen FOD 11:50

Nogar, Nicholas ThPC 109
Nogar, N. S. WPI 291, WPD 083

Noggle, F. T. TPE 206

Noguchi, Kazuyoshi MPF 257, MPF 256

Nold, Michael ThPA 006

Noon, Kate TOC 05:00

Noon, Kathleen R. ThPA 044

Norberg, Peter WPC 075

Nordhoff, E. TPF 219

Nordmeyer, Trent MPD 146

Norwood, Curt TPF 223

Norwood, Daniel TPB 054

Novikova, Ludmila I. MPB 065

Nugent, Kerry TPC 117, WPG 178

Nuwaysir, Lydia MPF 247, TPF 242, TPG 284

Nyberg, Fred MPF 243

O'Connor, Peter B. MOC 04:20

O'Donnell, Niall ThPE 152

O'Hair, Richard A. J. TOA 10:30, ThPA 011

O'Shannessy, Daniel ThOB 04:00

O. Marques, M. Graa MPA 044

Oberth, C. H. TPD 128

Obukowicz, Mark ThPD 137

Oda, Robert P. FOB 11:10

Odom, Robert ThOD 05:00, ThOD 11:30

Ogan, Kenneth ThPC 105, WPG 182

Ogawa, Tomoya ThPE 159

Oglesby, Thomas WPH 227, WPG 173

Oglesby, Thomas D. MPF 245, FOC 11:10, WPG 175, TOA 04:20

Ogorzalek Loo WPI 246, MPE 204, MPE 203

Oh, Chang-Hwan TPF 227

Ohashi, Mamoru ThPE 171, TPG 274

Ohashi, Yoko ThPE 159

Ohorodnik, Susan WPF 132

Okuyama, Tsuneo MPF 275, TPG 253, TPG 262, ThPG 296, MPF 274

Olberding, Edward MPD 189

Olberding, Edward L. MPD 188

Olivares, Jose MOF 04:20

Olivares, Jose A. WPD 076, MOF 05:00

Oliver, Ronald W. A. ThPG 288, ThPG 252

Olson, Douglas TPB 099

Olumee, Zohra ThPF 238

Omori-Satoh, Tamotsu TPG 262, MPF 275

Onigbinde, Adebayo MPA 028

Orians, Kristin J. WPA 010

Orkiszewski, Ralph TPA 008, TPA 048

Orlando, R. ThPE 156, ThPE 158, ThPE 157, MPF 264, MPF 261

Osborn, Blaire L. WPG 194

Ospina, Maria ThPD 140

Ostrovskaya, Galina MPF 252

Otsuka, K. WPD 081

Ott, Istvan MPF 273

Ottinger, Sean E. TPD 137

Overberg, A. TPG 259, WPI 275

Overney, Gregor WPB 041 
Overton, Santford MPC 109, MPB 071

Owens, Gary TPG 247

Owens, Kevin MPB 095, ThPF 224, WPI 282, WPI 272, ThPF 223, WOD O4:00, MOC 10:30, WPI 278

Owusu, Daniel ThPA 008

Painter, Sherry TOF 11:30

Paiva, Tony MPE 214

Paleos, C. M. ThPA 042

Palmer, Cynthia A. FOC 11:30

Palmer, Peter T. WPF 147

Pan, S. MPF 264

Panagiotou, Thomai WPF 152

Panico, Maria TOD 11:30

Pannell, Lewis ThPG 262, ThPG 261, TPF 218, TPF 236

Papac, Damon ThOB 04:20, WPI 239

Papaleo, Ricardo ThPA 035

Papayannopoulos, Ioannis A MPF 246, MPF 270

Papov, Vladimir MPF 266

Pappin, Darryl TPG 264

Pardoll, D. ThPG 280

Parees, David ThPA 029, WPI 278, MOC 10:30

Park, Frederick D. S. FOE 11:30

Park, Hyeung-geun TPE 152

Park, Melvin ThPF 194, ThPF 193

Park, SungAe MPF 260

Parker, Mark A. ThPB 054

Parker, Carol WPI 239, ThOB 04:20

Parlar, H MPD 197, ThOF 11:10

Parry, David. E. MPA 002

Pasa-Tolic, Ljiljana ThPB 065, ThPF 200, ThPA 005, ThPF 201, MPA 046

Pastel, Michael MPC 139

Pastor, Salvador J. TPA 043, ThPF 203

Pastor, Steve MPE 235

Pastorova, Ivana WPC 052

Patkin, Adam WPB 049

Patrick, Jeffrey ThPA 010, FOE 11:10

Patterson, Dale ThOD 11:10

Patterson, Donald G., Jr. ThOF 09:50, TOB 04:20, TPD 140, MPD 168

Patterson, Kathleen S. WPF 140, WPF 141

Paul, G. J. C. ThPA 042, TPF 245

Paulino, Jose MPA 001

Paulus, Gert ThPF 234

Pawlosky, Robert ThPD 143

Pearson, Paul TPD 139

Pearson, Paul G. WPG 188

Pearson, Richard WPF 147

Pearson, Roger ThOF 10:30

Pedder, Randall E. ThPB 048, ThPB 047

Peiris, Dil ThPA 009

Pellascio, Bernhard MPF 280

Peltier, John TPA 013

Penn, Sharon G. ThPB 064

Penn, Sharron G. MPE 205

Pepinsky, R. Blake MPF 270
Pereira, Neila ThPA 036

Perez, James ThPB 072

Perkins, John WPG 168

Perkins, John R. ThPG 269

Perreault, Helene ThPE 163

Perry, J. FOA 09:30

Peru, Kerry M. MPD 183

Pesch, R. MPE 217, MPE 218

Peschke, Michael TOF 04:00, MPA 036

Peter-Katalinic, Jasna ThPE 161, ThPE 160

Peterman, Scott MPA 027

Peters, N. K. ThPE 158

Petersen, Mary WPG 184

Peterson, Charles M. ThPG 294

Peterson, Kent MOB 10:30

Petitou, Maurice MPE 228

Petrosyan, V. S. WPF 139

Philip Wong, MPA 007

Philippe, Lihre ThPC 107

Phillips, Andy TPB 110

Phillips, D. H. TPD 138

Phillips, Eugene MPD 150

Phillips, Lawrence R. MPC 123, WPG 197

Phillips, Mark A. TPE 188

Picard, Claudine MPE 228

Picotte, Pierre TPD 149

Pierce, Richard H. TPD 134

Pincock, J. A. MPC 112

Pinkston, J. David TPB 096

Pires de Matos, Antsnio WPI 293, WPA 011

Pirhalla, Jill WOA 11:50

Pitsenberger, Cynthia WPE 110, ThPB 060

Plante, Sylvie TPF 220

Pleasance, Stephen TPE 187, WPG 187

Plomley, Jeffry B. MPD 199, ThOC 05:00, ThOF 11:30, ThOF 11:30

Pogue, Robert WPC 073, ThPA 004

Pogue, Robert T. MPC 130, MPC 129

Poisson, L. ThPB 050

Pokrovskiy, Valeriy MPF 252, WPC 074

Polce, Michael J. TPA 022, ThPA 013

Pomerantz, Steven C. TPF 237

Ponciano, Cassia ThPA 036

Poon, G. K. TPD 138, TPE 153

Poon, Grace K. TPE 162

Pope, Marshall TOC 09:50, MPA 004, TOF 12:10

Potier, Noelle TPF 232

Poulsen, Fleming MPE 206

Poutsma, John MPA 001

Povey, William T. MPB 0053

Powell, David ThPD 140

Powell, Mark WPG 202

Powell, Mark L. WPG 179

Powers, Daniel D. MPF 251

Powis, Simon ThPG 281

Poyer, J. Lee ThPD 147

Pozniak, Boguslav P. TOF 11:50

Prabhakaran, Muthu TPF 230

Pradeep, Thallapil FOE 11:10 
Pragnell, Charlton MPD 162

Prakash, Chandra TPE 197, TPE 176

Pramanik, B. MPF 262

Praphairaksit, Narong MOF 12:10

Prather, Kimberly MPD 149, MPD 147, MPD 146, MPD 148

Preece, Steve TPE 169

Prell, George D. TPF 243

Preston, Andrew WPB 043

Price, John M. WPB 032

Price, Keith R. ThPE 168

Prockop, Darwin TPF 238

Proctor, Andrew TPE 199, WPC 054, FOB 11:50, ThOD 10:30, TPE 184, WOA 04:20, WOA 04:40

Prokai, Laszlo MPF 241, MPE 222, MPE 226

Prokai-Tatrai, Katalin MPF 241

Prom, Jean-Claude MPF 284

Prome, Jean-Claude MPE 228

Prospero-Garcia, Oscar ThPD 126

Provost, Lionel $R$. MPE 236

Przybylski, Michael TPG 256, FOD 11:50

Ptillot, Yves ThPE 153, MPF 278

Pucci, Sergio WPF 156, MPC 125

Pullen, Frank TPB 062

Pungor, E., Jr. ThPE 151

Purdon, Michael P. TPD 143

Puretzky, A. A. ThPF 220

Pureveen, Jos WPC 051

Purves, Randy WPH 204

Pykdldinen, Mika MPA 030

Pyle, Steven ThPC 123

Pylypiw, Harry, Jr. MPD 182

Pyne, David A. WPH 220

Pyrek, Jan St. MPC 130, MPC 129, MPC 118

Qian, Kuangnan WPI 285, MOC 04:20, WPC 067

Qian, Mark G. TPG 251, TPF 214, TPG 292, FOB 11:30, TPB 074, WPI 260

Qin, Jun ThOC 12:10, ThPC 081

Qu, Yun TPA 030

Quarmby, Scott T. ThPC 100

Quirke, J. Martin E. ThOE 09:30, ThOE 10:10

Rabinovich, Yakov I. FOB 11:50

Rachel, R. WPI 246, MPE 204, MPE 203

Radford, Sheena ThPG 255

Radom, Leo MOB 09:30

Raffaelli, Andrea MPC 125, WPF 156

Ragsdale, John ThPC 096

Raida, Manfred TPG 248, MPF 254

Rainbird, B. J. MPD 187

Rajamani, Umamaheswari ThPG 261, ThPG 262

Ralitsch, Milan ThOF 11:50

Ramadas, Bhat MPE 214

Ramaley, Louis I-2 FOB 10:30

Ramanathan, Ragulan MPE 222

Ramesh, Rao MPD 179
Ramirez, Jackie WPG 182

Ramsey, J. Michael MPC 115

Ramsey, J. M. WPD 086

Ramsey, Roswitha S. TPB 088

Ranasinghe, Asoka TPD 126

Ranasinghe, Yasmin A. MPB 060, MPA 040

Randall, Linda L. ThPG 251

Rappmund, Andreas MPA 020

Raptakis, Emmanuel MPB 089

Ratain, Mark WPG 182

Ray, March ThPC 107

Ray, Kenneth MPD 191, TPB 084

Rebek, Julius, Jr. TPC 123

Reddick, Christopher WPI 270

Reddy, D. Meghanath(1) TPF 243

Reddy, G. R. TPF 229

Redpath, Craig WPI 280

Reed, Don TPD 125

Reed, R. H. FOB 12:10

Reed-Bombick, Betsy ThPG 291

Reents, William D. ThPB 067, ThPB 071

Reeve, Joseph, Jr. MPF 242

Regert, M. WPC 053, WPG 193

Regg, Brian TPB 071

Regnier, Fred ThOD 11:10, TPB 070

Reiber, Duane ThPF 235, WOD 10:30

Reich, Fraser ThOD 10:10

Reichlmaier, Stefan MOC 11:10

Reilly, James ThOD 04:40, ThOF 04:00, MPB 086, TOD 04:00, ThPF 229

Reinhold, Bruce B. ThPE 165, ThOB 10:10

Reinhold, Vernon ThPE 165

Reinhold, Vernon N. ThOB 10:10

Reiter, Alex MPF 249, MPF 250, TOC 10:10

Remberg, Barbara MPC 101

Rempel, D. L. WPE 098

Requejo, Adolfo MPD 164

Resch, Martin ThPF 234

Rettenmeier, Albert W. WPG 170

Reuschel, Scott A. TPE 208

Reynolds, David J. WPB 033

Rhomberg, Andrew ThPE 174

Rich, Jennifer MPA 024

Rich, Michaela MPA 027, MPD 150

Richards, Don TPB 062

Richards, James I-2 ThOB 11:30

Richardson, D. E. WPA 008

Richardson, Susan TOB 10:30

Richardson, Susan D. WPF 140, WPF 141

Ridder, Gregg M. TPC 114

Ridge, D. P. MPA 028, FOA 11:50

Ridge, Douglas MPA 047

Ridge, Douglas P. WPI 285

Rieger, Robert A. TPF 240, TPF 243

Riggs, B. Lawrence FOB 11:10

Rimando, Agnes TPB 056

Rindgen, Diane TPF 226

Rinehart, Kenneth ThPG 290, ThPG 275

Rippy, Allyson WPA 019 
Riveros, Jose M. ThPA 009, TPA 024, MPA 017

Robbins, Ronny ThPA 020, MPC 116

Robbins, Winston ThOE 05:00

Robert, Croes MPD 181

Roberts, Gerald TPG 258, TPC 122

Robertson, Duncan H. L. MPF 259

Robertson, Philmore TPE 198

Robinson, Carol WOC 04:40, MPE 206, ThPG 255

Robinson, J. M. MPB 090

Robinson, Kevin WOD 05:00

Robke, David TPB 051

Roboz, John MPE 215, MPF 273

Rockwood, A. L. MPB 084, WPH 229

Rockwood, Alan ThPB 073

Rockwood, Alan L. WPE 112, WPE 114

Rockwood, Alan W. WPB 032

Rodebaugh, Ron MPE 235

Rodgers, M. T. WPA 001

Rodrigez-Cruz, Sandra TOC 11:50

Rodriguez-Cruz, Sandra TOC 04:00, MPA 043

Roehling, Ulrich ThPF 204

Roepstorff, P. TPF 219

Roepstorff, Peter MPF 283

Rohde, Michael ThPG 263

Roher, Alex MPF 240

Rohrbaugh, Dennis MPD 186

Rohrs, Henry FOE 11:10

Rolando, C. ThPA 026, ThPB 049, ThPB 050, WOB 11:30, WPH 211, WPC 053, WPA 007, WPG 193, WPA 012

Romeo, Elena ThPD 146

Rontree, John WPF 138

Rosazza, J. P. N. TPD 146

Rosell, Joan WOB 12:10, MPB 058

Roskey, Mark TOD 04:20

Rosnack, K. J. FOC 11:50

Rosnack, Kenneth MPF 281

Ross III, Charles MPA 046

Ross, Andrew R. S. WPA 010

Ross, Mark MPF 267

Ross, Mark M. ThPE 170

Ross, Philip ThPA 012

Rossi, Vronique ThPE 153

Rourick, Robyn MPC 107

Rourick, Robyn A. TPE 202, TPB 061, TPC 119

Rouse, Jason C. TOD 11:10

Rousseau, Caroline MPC 138

Roussis, Stilianos G. WPC 072, WPC 071

Roy, Laura ThPB 080

Roy, Raymond ThPB 080

Roy, Tim WPF 150

Roybal, Jose WPG 189

Rudensky, Alexander Y. ThPG 283, ThOB 12:10

Ruiz, Migdalia MPC 111

Russell, David TPA 006, WPB 030, WOD 04:20, WPE 118, WPI 242, ThPF 228

Russell, David H. ThOA 04:00, WOD 11:30, WPH 226, ThPF 232, ThPG 256, TPB 073, ThPA 039, ThPF 236
Russell, David J. MPC 105

Russell, John W. WPG 183

Russell, K. C. MPE 220

Rybal'chenko, Vladimir MPF 252

Ryzhov, Victor MPA 010

Sablier, M. ThPA 026, ThPB 049, WPA 007, WPH 211, WOB 11:30, WPC 053, ThPB 050, WPG 193, WPA 012

Sackett, Danny ThPG 261

Sadeghialavijeh, Mehrnoosh ThPF 227

Sadilek, Martin WOE 11:30

Sadoun, Aladin MOB 04:00

Sadovskaya, Irina I-2 ThOB 11:30

Saegesser, Martin MPE 227

Saha, Sanat TPG 261, ThPG 249

Sahlstrom, K. E. TOF 10:10

Sakairi, Minoru WPH 203, TPB 080

Sakuma, Takeo MPE 221, MPD 190, TPD 149, TPE 179

Sakuma, Takeo I-2 ThPB 051

Salat, Janos MPF 273

Saleh, Samir A. WPG 199

Salem, Norman, Jr. ThPD 136

Salem, Norman ThPD 143

Salt, Kimberly MPD 147

Salt, Kimberly MPD 148, WPF 156

Salyan, Mary Ellen K. ThPE 162

Samama, Jean-Pierre MPF 284

Samuel, Mitrovich TPC 124

Sanaullah, TOA 05:00

Sanchez, Gabriel TPD 149, TPE 179

Sanchez, Stephanie C. WPG 199

Sander, Peter WPB 031

Sanders, Mark MPC 133

Sandersson, Katarina MPF 243

Sang, Hong ThPD 147

Sannes, Kristin WPI 264

Sarkisian, Molly TPF 241

Saru, F. WOB 11:30, WPH 211

Sarver, Emory W. ThPC 090

Sasaki, Tania A. TPB 108

Sasmor, Henri ThPG 259

Sassen, Roger MPD 164

Satsangi, Neera TPE 174

Satzger, Duane TOA 11:50

Satzger, R. Duane TPB 092, TPD 144

Saulys, Dovas A. MPE 208

Saves, Isabelle MPF 284

Sawada, Masami MPA 049

Sayer, Jane TPF 236

Scarberry, R. E. TPG 285

Schaaff, Thomas TPA 030

Schachterle, Steve TPB 104, TPB 105, TPB 106

Schaefer, William TPB 050

Schaeffer, Richard ThPB 046

Schaeffer, Richard A. ThPB 048, ThPB 047

Schaer, Martin WPI 273 
Schaffter, L. M. MPC 106

Schaller, Johann MPF 280

Schaps, Marlene E. TPB 103

Scheller, Nova TPD 126, TPD 127

Schenkel, Thomas FOE 11:50

Scherer, Stefan MPB 087

Schey, K. L. TPG 273, MPF 287

Schey, Kevin L. FOE 09:30

Schieltz, David TPG 279, MPF 294

Schieltz, David M. TPG 278

Schindler, D. W. ThOF 11:10

Schlunegger, Urs P. ThPB 057, MPF 280, ThPF 237, MPD 141, MPD 142, MPB 080

Schmehl, Russell H. TPB 079

Schmidt, E. G. WPE 102

Schmidt, Joseph A. R. TOA 10:30, ThPA 011

Schneider, Dieter H. FOE 11:50

Schneider, Joanne MPD 178

Schneider, Klaus TOD 11:50

Schneider, Rick WPG 167, TPE 213

Schnier, Paul TOC 04:00

Schnier, Paul D. WPH 224

Schnier, Paul S. TOC 11:50

Schnolzer, Martina MPF 293, TPG 270

Schorno, Karl S. WOA 12:10, WPG 181

Schrader, W. MPE 218, MPE 217

Schreurs, Jolanda MPF 263

Schriemer, Dave C WPI 277, WPI 245

Schubert, Ralph ThPE 187

Schubert, Michael TPB 086, ThPC 088, ThOC 11:10, ThPC 087

Schuerch, Stefan MPD 142, MPD 141

Schuhmacher, Martina TPG 256

Schultz, Birgit MPF 282

Schumaker, Verne ThPE 153

Schurz, Helen TOB 11:50

Schuyl, P. J. W. ThPD 125, WPC 064

Schwartz, Brenda WOC 05:00, WPE 115

Schwartz, Brenda L. ThPG 251, TOC 04:40, MOD 05:000

Schwartz, Jae ThPC 095, ThPC 096, ThPC 097, ThPC 084, ThPC 094, MPC 120, WPG 178, ThOC 04:40=

Schwartz, Jae C. ThOC 11:50, ThPC 098, MPF 245

Schwartz, Jay TPE 151, WPG 165

Schweighofer, Andreas MPA 026

Schweikert, Emile ThPA 037, ThPA 034, WPD 089, ThPA 038

Schweikhard, L. WPE 123, WPA 002, WPI 290, WPE 124, WPE 122

Schweikhard, Lutz WOC 04:00

Schweingruber, Hans MPE 211

Scoble, Hubert WOD 11:10

Scoble, Hubert A. TOD 11:10

Scott, Gary MPB 097

Scrivener, Elaine WPB 033

Scrivens, Jim WPC 066, MOC 10:10, WPC 062, WPC 063, WPC 057, WPC 056

Scullion, Simon WPF 157

Sears, L. J. ThPD 145
Sebo, Peter ThPG 274

Seedorf, Ronald WPF 142

Segal, John MOC 10:10

Selzer, J. ThPG 270

Sen, Atish MPA 039

Sena, Marcelo TPA 024

Senko, Michael W. WPB 040, WPE 119, ThPB 065

Sepetov, Nikolai TPC 113

Sericano, Jose MPD 177, MPD 165

Serrano, Jose TPD 147, MPD 167

Seta, Kazuo TPG 253, MPF 274, MPF 275, TPG 262, ThPG 296

Severinov, Konstantin MPF 255

Severinova, Elena MPF 255

Seyfreid, Thomas N. ThPE 165

Shabanowitz, Jeffrey ThPG 286, ThPG 282, ThPC 285 , TPG 247, ThOB 11:50, ThPG 274, ThOB 10:30, TPB 069, TPG 264

Shackleton, Cedric H. L. ThPG 295

Shaffer, Scott A. ThPA 025

Shaffer, Barrie A. ThPC 093

Shaffer, Scott MOB 04:40

Shafter, Elizabeth MPD 195

Shah, Bhavana ThPE 172

Shah, Nikhil WPH 231, TPC 116

Shahgholi, Mona ThPE 170

Shalaby, Lamaat WPI 247

Shaler, Thomas WPI 264, WPI 263

Sharp, T. R. MPC 124, FOB 12:10, FOC 11:50, TPA 012

Shartava, Archil ThPG 264

Shaw, Angela TPB 099

Shaw, R. W. WPD 086

Shay, Brian J. MPC 119, WPC 064

Shealy, Dana B. MPD 194

Sheehan, Edward WPH 215, WPH 216, WOB 04:40

Shen, Nanzhu TOF 12:10

Shen, Shida MPB 059

Shen, Tun-Li ThPG 289

Sheng S. Yang, MPA 007

Sheppard, Robin MPC 110, MPB 061

Sherman, M. G. WPH 229

Sherman, Michael G. ThPB 066

Sherman, Nicholas E. TPG 264, ThOB 10:30

Sherman, Paul MPC 122

Shevshenko, Andrej FOB 09:30, TPG 276

Shew, Sandy TPA 041

Shick, Charles, Jr. MOC 04:40, MOF 11:50

Shiea, Jentaie WPH 233

Shieh, Fang-Jir MOF 11:30

Shieh, J. J. ThPD 137

Shimada, Ichio MPF 257, MPF 256

Shinn, David ThPF 197

Shofstahl, James FOC 09:50

Short, R. T. WPD 082

Short, Robert ThPB 045

Shrader, S. ThPB 079

Shu, Chi-Kuen TPB 102

Shuguang Ma, MPA 007

Shukla, A. K. TPA 029 
Shukla, Anil K. TPA 028

Shum, Sai MPE 235

Shushan, Bori WPH 225, MPD 190, TPD 149, MPE 221, TPE 179, ThPB 051

Shyong, Ban-Jen WPI 249

Sible, Emily M. ThPE 185

Sickenberger, Dave W. ThPD 141

Siegel, Marshall MPF 277, MPC 139, MPF 276, ThPG 279

Siethoff, C. MPE 218

Silverton, Susan F. ThPC 115

Silvestro, Luigi ThPE 175

Sim, P. Greig MPD 159

Simanek, Eric E. WPH 232

Simay, Antal MPF 273

Simon, Jerald TPB 079

Simonneau, G. ThPB 049

Simpson, J. T. TPB 101

Sims, Gary R. ThPB 052

Singh, Padma MPE 211

Singh, Rajendra. K. WPH 236

Singh, Romi ThOC 04:40

Singleton, David MPF 281

Sirimanne, Sarath MPD 168

Sirimanne, Sarath R. TPD 140

Sisenwine, S. TPE 166

Sisenwine, Samuel WPH 227

Sisk, Ellen C. TPF 234

Siu, K. W. Michael WPF 164, WPH 225, WOB 05:00, TPA 004

Siuzdak, Gary WPH 207, ThPD 126, MPF 258, TPF 241

Sjvvall, Jan TPG 268, ThPD 144

Skarping, Gunnar WPG 198

Skeen, Todd T. WPF 149

Skipper, Jonathan A. ThOB 11:50

Skipper, Jonathon ThPG 285

Slawson, Matt H. TPE 208

Slayback, John R. B. TPB 059, MPC 105, MPC 102

Slingluff, C. L. ThOB 11:50

Smelyanskiy, Yanina MPE 203

Smirnoff, Igor TOD 04:20

Smith, David L. MPF 265

Smith, Brian WPB 028

Smith, Claudia A. TPB 096

Smith, D. H. WPD 086

Smith, David H. MOF 09:50, WPD 077, WPD 078

Smith, David L. FOD 11:30, ThPG 250, ThPG 247, TPB 068, MPB 096

Smith, Diana M. TPG 255

Smith, Douglas A. ThOA 11:10

Smith, Glenn ThPE 184

Smith, Jacob D. ThPA 011

Smith, Jean B. TPB 068, MPF 265

Smith, Philip C. TPE 190

Smith, R. D. WPH 229, MPB 084

Smith, Rebecca MPA 023, MPA 022

Smith, Richard ThPB 073, WPE 115, WOC 05:00
Smith, Richard D. TPB 077, MOD 05:000, TPF 233, WPE 117, MPE 213, WPE 112, TPB 085, TPF 228, WPH 232, FOD 10:10, ThPG 251, WPE 114, TOC 04:40, TPF 234, ThPB 066, TPB 087, WPE 113, TOC 10:30, TPA 001, WPH 223, TPE 154

Smith, Steve WPB 043

Smith, Virginia F. ThPG 251

Snider, C. E. MPF 288

Snider, Neil S. WPG 185

Snyder, A. Peter TOF 04:20, ThPA 031, ThPA 030

Sochacki, Marek TPF 235

Soloski, Mark ThOB 11:10

Solouki, Touradj MPA 046, ThPF 201, ThPF 202, WPE 118, WPA 018

Soloveytchik, Boriana WPI 283

Solsten, R. Thomas MPE 212

Somani, H. TPE 153

Somogyi, Arpad ThPA 028, TPA 015, TPA 045, FOE 10:10, TPA 044

Song, Siqing MPF 285

Song, Ye ThPE 165

Soni, M. H. ThPC 111

Sorensen, Daniel MPA 016

Sorokine, Odile MPF 268

Souders, C. ThPE 151

Sovocool, G. Wayne TOB 05:00, MPD 202

Spalding, Roy MPD 175

Spanbroek, Rainer ThPE 160

Sparkman, David WPB 035

Sparkman, O. David MPD 145, WPF 134

Speakman, Alison WPG 198

Spear, Kerry L. TPC 117

Speir, J. Paul MOC 12:10, WPH 205

Sphon, James A. FOC 10:10

Spicer, V. ThPF 205, WOD 10:50, MPB 085

Spier, J. Paul WPH 206

Spiess, Iris WPE 097, ThPB 069, MPA 021, WPE 094

Spiess, Joachim ThPG 248, MPF 289, ThPA 022

Spik, Genevive ThPE 153

Spreen, R. C. MPC 106

Springer, David L. MOD 11:30, MPF 272

Sprinzl, Mathias FOD 11:50

Squires, Robert WOE 11:50, ThPA 007, MOB 05:00, MPA 001, MPA 013

Srinivasan, Jannavi R. WPI 265

Srinivasan, N. MPC 112

St-Germain, Frangois WPF 151

St. Claire, Bob WPG 165

Stacey, Catherine WPH 206, TPB 086, WPH 205

Stack, Robert ThPF 193

Stadlmann, Jens MPB 098

Stafford, G. ThPC 111

Stafford, George ThPC 095, ThPC 095, ThPC 084, ThPC 098

Stahl, Bernd ThPE 169, ThPE 160

Stahl, Bernt WPI 247

Stahl, Douglas C. WPB 026

Standing, K. G. ThPF 192, WOD 10:50, ThPF 205, ThOD 09:30, FOD 10:30, MPB 085 
Standing, Kenneth G. ThPG 249

Starrett, Anne TPB 060, MPC 107

Stavinoha, William B. TPE 174

Stayton, Patrick S. ThPG 251

Ste-Marie, Line TPE 164

Steenwyk, Rick C. WPG 188

Stein, Ronald ThOD 04:20, ThOD 04:00

Stein, Stephen MPC 100

Stein, Stephen E. WPB 036

Steiner, Robert MOF 10:10

Stemmler, Elizabeth WOC 11:30

Stephane, Bouchonnet ThPC 107

Stephanson, Niclas TPE 209

Stephenson, James L. TOF 10:30, TPA 035

Stern, G. A. ThOF 11:10

Stern, Gary MPD 197, MPD 193

Steup, Martin ThPE 169

Stevens, K. L. TOB 11:30

Stevens, Robert MPE 209

Stevenson, Tracy I. WPI 258

Stewart, Jon D. MPF 258

Stiller, Steven W. ThPC 105, WPG 182

Stillwell, W. G. TPD 129

Stockton, Brian MPA 025

Stoeckli, Markus MPB 080

Stoffel(s), J. J. MOF 04:40

Stoker, Paul W. ThPG 247

Stoney, Ken WPG 178, TPG 290, TPG 249

Stout, Howard TPA 041

Stracke, Patric MPB 098

Stramare, Luca WPF 144

Streeper, Rob TPD 139

Streeper, Robert T. TPE 190, TPE 174

Street, Jacqueline ThPE 163

Stresau, Dick ThOD 12:10

Stresser, David M. TPD 142

Strife, Robert ThPC 094, MPC 120

Strobel, Fred TOC 10:10

Stroh, Justin MPF 281

Stromatt, Robert MPE 233

Stroobant, Vincent ThPD 124

Stroud, Mark R. ThPE 162

Strupat, Kerstin WPI 257

Strupp, Christian MPF 258

Stults, John WOC 11:10, MPF 271, MOD 10:30

Subramanian, Periyasamy TPB 100

Suleiman, Ahmad A. MPE 223

Sullards, Cameron MPE 230, TPA 003

Summerfield, Scott MPF 248, ThPG 292, WPD 091

Sumner, Lloyd ThPF 228

Sumner, Lloyd W. ThPA 039, ThPF 236

Sun, Furong ThPG 290

Sun, Y. MPF 288, TPG 252, TPA 042

Sundqvist, Bo ThPF 231, ThPA 035

Sundqvist, Bo U. R. TPA 040

Sunner, J. MPF 279

Sunner, Jan WPI 255

Supko, Jeffrey G. MPC 123, WPG 197

Sutton, C. TPG 259, WPI 275
Sutton, C. W. TPG 254

Sutton, S. W. ThPE 150

Suzuki, Sakaru ThPE 171

Swackhamer, Deborah L. ThOF 10:30

Swanek, Frank D. TPB 087

Swanson, Steve TPE 158

Swedberg, S. ThPE 151

Swenberg, James A. TPD 126, TPD 127

Swenson, David MPE 211

Swiderek, Kristine M. WPB 026

Syka, John ThPC 095, ThPC 098, ThOC 11:50

Szekely, Gabriella ThPF 215, ThPF 216

Szulejko, Jan ThOA 10:30

Tabei, Keiko ThPG 279, MPC 139, MPF 277, MPF 276

Tachiyashiki, Satoshi MPE 229

Tagami, Uno MPF 257, MPF 256

Taghizadeh, Koli TPB 049

Takach, Ed TOD 04:20

Takada, Yasuaki TPB 080, WPH 203

Takai, Yoshio MPA 049

Takayama, Mitsuo ThPA 024

Talaga, Philippe ThPE 169

Talrose, Victor L. MPB 065

Tam, Steve TPF 235

Tanaka, Koichi ThPF 234

Tang, Dazhi MOD 10:10

Tang, K. MPF 237, WPI 259

Tang, Kai TPF 216

Tang, Liang ThPB 063, WOB 10:30

Tang, Ming ThPC 119

Tang, Wei TPE 156

Tang, $X$. ThPE 154, MOC 11:30

Tang, Xiaodong WPI 266

Tang, Xue-jun ThPG 249, ThPG 258

Tang-Liu, Diane TPE 201, WPG 166

Tannenbaum, S. R. TPD 129

Taranenko, N. I. ThPF 220, WPI 259, MPF 237

Tarr, George ThOD 11:10, TPB 070

Tata, Prasad TPE 184, TPE 199

Tayler, Paul WPC 066

Taylor, Dennis ThPC 084, ThPC 098, ThPC 096, ThOC 11:50, ThPC 095

Taylor, Lester ThOC 04:40

Taylor, Mike WPC 062, MOC 10:10, WPC 066

Taylor, Paul TPC 122

Taylor, Paul A. MPF 245

Taylor, Steven MPF 267

Taylor, Susan TPB 060, TPG 249, MPC 107

Taylor, William WPA 019, WPA 020

Tchekhovskoi, Dmitrii V. WPB 036

Tedeschi, Nicole ThPG 266

Teesch, Lynn TPD 146, TPB 100

Teklehaimanot, Bibi TPA 026

Teng, Siu ThOE 11:50

Termin, Andreas ThPE 172

Terunuma, Hideya TPF 238

Terwilliger, Thomas C. FOD 10:10 
Thberge, Roger ThPA 041

Thanabal, Venkataraman MOD 12:10

Theophanides, T. TPF 245

Thibault, Pierre ThPB 051, ThOB 11:30, ThPE 153

Thibault, Pierre I-1 FOB 10:30

Thierry, Blasco ThPC 107

Thilens, Nicole ThPE 153

Thomas, Angela MPD 185

Thomas, Boban TPF 243

Thomas, Craig ThPA 004

Thomas, D. W. ThPD 145

Thomas, Darren MPD 159, ThPG 273

Thomas, Stan W. ThPC 120, ThOC 04:20

Thomas, Susan WPD 088

Thompson, Cyril WPF 146

Thompson, Harold C. TPB 095

Thompson, Stephen WOD 05:00

Thompson, Toni TPE 178

Thompson, William MPC 107

Thompson, William L. MPC 134, TPB 061, MPC 102

Thomson, Michael L. MPC 111

Thorne, Jacqueline MPB 063

Thorne, Peter S. TPB 100

Thorpe, Colin MPA 047

Thrall, Karla ThOF 04:40

Thruston, Alfred D., Jr. WPF 140, WPF 141

Tianlan, Zhang WPI 285

Tippin, Tim WPG 165, TPE 151

Tobe, Yoshito MPA 049

Tobias, Herbert J. MOF 04:00

Todd, John F. J. MPB 0053, ThPC 086, WPB 042, MPD 153, MPB 054, MPC 128, MPA 018, MPA 037

Todd, Peter ThPB 045, ThPE 176, ThPA 043, ThPD 138

Tomany, Michael TPG 250

Tomer, Kenneth ThOB 04:20, WPI 267, WPI 239

Tomlinson, Andy J. TPG 267, FOB 11:10, TPG 286, TOA 10:10

Tomlinson, John TPD 144, TOA 11:50

Tomy, Gregg MPD 193

Tong, Huayi MPE 235

Torok, D. S. TPB 101

Torres, T. A. WPE 101, WPE 103

Tosh, R. E. TPA 028

Townsend, Jennifer A. ThOF 11:30

Tran, Huu M. TPG 255

Travers, Jeffrey ThPD 131

Tretyakova, Natalia MPC 131

Tria, John WPC 075

Trimble, Laird TPE 167

Triolo, Antonio TPE 186

Truong, Long ThPE 148

Tsao, Rushung MPC 139

Tseng, Jih-Lie MPF 244

Tsuchiya, Masahiko MPB 064

Tsuneyoshi, Toshihiro TPF 238

Turecek, Frantisek WOE 11:30, ThPA 019, MOB 04:40, MPE 231, MPE 232, WPH 228, ThPA 025

Turesky, R. J. TPD 129
Turesky, Robert J. TPF 226

Turner, Wayman E. TOB 04:20

Turnipseed, Sherri WPG 189

Tykodi, Scott ThPG 285

Uchida, Kazuhisa ThPG 296, MPF 274

Udiavar, S. ThPE 151

Udseth, H. R. MPB 084, WPH 229

Udseth, Harold R. TPB 085, ThPB 066, TPF 234, WPE 114

Uhrich, Mark TPE 151, WPG 165

Uhrich, Mark D. MPF 245

Uhrig, Mary ThPG 291

Unanue, Emil R. ThPG 287

Urban, Jan TPG 269

Uzabiaga, Frangoise MPE 228

Vachet, Richard TPA 048

Vachet, Richard W. TPA 011, ThPC 092, ThPF 197

Vachon, Benoit WPF 151

Vachon, Luc TPE 164

Vadal', Gianni Giulio MPD 157

Vaidyanathan, Gopalakrishnan ThPC 122

Vainiotalo, Pirjo MPA 030

Vaisar, Tomas TPG 269, MPE 231, MPE 232

Valaskovic, Gary TPG 288

Valaskovic, Gary A. MOC 04:20

Valero, Gustavo WPF 147

Valiga, Robert E. WPD 077

van Baar, B. L. M. MPD 172

Van Berkel, Gary WPH 222, MOF 10:30, WPH 221, WOB 11:10

Van Berkel, Gary J. ThOE 09:30, TPB 088

van Breemen, Richard B. MPF 251, TPB 056, TPB 057

Van de Water, Judy ThPF 242

Van den Heuvel, Hilde ThPE 166, ThPE 168

van der Doelen, Gisela WPC 051

Van Dorsselaer, Alain TPF 232, MPE 220, MPF 268, MPE 219

van Grevenstein, A. WPC 051

Van Hoof, F. WPF 138

van Koppen, P. A. M. FOA 09:30

van Kuijk, F. J. G. M. ThPD 145

van Ocht, J. WPC 051

Van Orden, Steven TOC 10:30, ThPB 066, WPB 032

van Rooij, Gerard J. WPE 126

van Soest, Remco TPE 207

Van Stipdonk, Michael ThPA 038, WPD 089, ThPA 037

van Vyncht, Gery TPE 169

Van Ysacker, Peter G. TPB 110

Vartanian, V. H. WPE 107, WPE 100, WPE 103

Vartanian, Victor ThPC 119, ThOA 12:10

Vasconcellos, M. A. ThPD 125

Vath, James E. TOD 11:10, WOD 11:10

Vatsadze, Sergey MPC 131

Vaughan, J. ThPE 150 
Velupillai, Palanivel ThOB 10:10

Venesky, John S. MPC 134

Venkataramanan, Raman TPE 199, TPE 184

Venot, Andre ThPE 172

Ventura, M. C. ThPC 108

Verentchikov, A. V. ThPF 205

Verentchikov, Analoti TPG 250

Vertes, Akos MOC 11:30, ThPF 238, ThPE 154, WPI 283, ThPF 227, WPI 244, WPI 266

Vestal, Marvin ThPF 195, TOD 04:20, TPG 250

Vestling, Martha M. TOD 10:30

Veverka, Karen A. TPE 177

Via, Jim TPB 097

Vidavsky, Ilan ThPG 287, TPF 222, ThPG 284

Viger-Chougnet, Antoinette TPE 172

Viggiano, A. A. WOE 10:50

Vigushin, D. TPE 153

Vincent, Gervat MPA 034

Viner, Nicholas J. ThPG 287

Vinogradov, Serge N. ThPC 288

Visentini, Josie MPC 138

Vlasak, Paul R. MPB 093

Vogt, Volker M. MPF 270

Volk, Kevin MPC 134

Volk, Kevin J. MPC 136, TPB 061, TPE 202, TPC 119

Vollmer, David TPD 131, TPD 132, TPG 260

Volmer, Dietrich TPB 091

Volmer, Dietrich A. MPD 169, MPD 170, MPB 074

von Helden, G. TPB 076

von Wittenau, Alexis Schach FOE 11:50

Voorhees, Kent ThPC 114

Voorhees, Kent J. MPB 074, ThPD 142, ThPD 141

Vorm, Ole ThPF 204, MPF 283, TPG 276

Vossbrinck, Charles ThPG 290

Vouros, Paul TPE 178, WPH 219, MPF 269, TPF 226, TPC 123, MPD 156, TPF 223, FOB 10:10, MPB 063, WPF 152

Voyksner, Robert WPI 271, TPB 055, ThPC 121, TPB 072

Voyksner, Robert D. MPE 216, WPF 155

Vrbanac, James TPD 139, TPA 048

Vulpius, Tore ThPA 003

Wachs, Timothy MPB 061, WPG 174

Waddell, Dave S. ThOF 11:30

Wade, Terry MPD 165, MPD 166, WPF 128, MPD 177

Wagner, David TPC 118

Wagner, David S. FOD 11:10

Wagner-Redeker, Winfried TPE 182

Wahl, Karen MPE 233

Wainhaus, Samuel B. FOE 12:10, FOE 10:30

Waite, Herb MPF 267

Wakefield, Michael MPC 133, WPB 048, TPB 064

Walker, Carthene B. ThPG 274, TPB 069

Walker, Kathleen L. TPB 090

Walsh, John TPE 151

Walther, C. WPE 124, WPI 290, WPE 122
Wanczek, Karl Peter MPA 019, WPE 095, ThPB 062, MPA 020, WPE 096, MPA 021, ThPB 069, ThPB 075, WPE 097, WPE 094

Wang, Bing TPF 217

Wang, Dingneng MPB 067, TPA 034

Wang, Fang ThPG 258

Wang, Guangdi WOB 04:20

Wang, HangQi TPB 070

Wang, Jia-shen WPB 029, MPC 121

Wang, Jianyao TPA 004, WOB 05:00

Wang, Jin WPF 163

Wang, Liping MPA 047

Wang, Mingda ThPC 102

Wang, Rong WPI 243, MPF 239

Wang, T.-C. L. TPB 093

Wang, Tao-Chin ThPD 136

Wang, Wei ThPG 285

Wang, Weimin WPH 214, WPH 209

Wang, Wen-shyang WPH 233

Wang, Xiaomin MPA 032

Wang, Yajun WPI 242

Wang, Yan TPD 148

Wang, Yang ThPC 087, ThOC 11:10, ThPC 088

Wang-Iverson, David WPG 183

Ward, Glen ThPD 136

Warrack, Bethanne TPG 249

Warshawsky, David MPD 178

Wasserman, Michael B ThPC 090

Watkins, Carl WPI 294

Watkins, Layle MPA 024

Watson, C. H. WPA 008

Watson, Cliff ThPB 068, MPE 226, MPA 035, TPG 275

Watson, Clifford TPA 014

Watson, Eric ThPE 173, ThPE 172

Watson, J. Throck TPE 210, WPI 254

Watts, Heath WPF 163

Watts, Peter ThPC 086

Waugh, Russell J. TOA 12:10

Wedge, P. MPC 112

Wei, Gang ThPF 190

Wei, Jian ThPB 046

Wei, Xiaona ThPG 256, WPH 226, TPB 073

Weil, C. ThPC 111

Weil, David A. TOD 05:00

Weinberger, Scot ThPF 196

Weinsotck, Joseph TPC 122

Weintraub, Susan T. ThPA 044, TPE 174, TPE 190

Weir Lipton, Mary S. MPF 272, MOD 11:30

WEISS, Christian WPG 200

Weissenberg, Klaus WPB 048

Welch, Kenneth J. WPF 153

Welch, Michael WOA 11:30

Wells, Greg TPB 107, ThPC 103, ThPC 104

Wells, J. Mitchell ThOC 10:10

Wen-Shyang, Wang WPH 234

Wendling, Jay MPC 122

Wensing, Michael W. ThPA 031, TOF 04:20, ThPA 030

Wenthold, Paul MPA 013, MOB 05:00 
Werdel, Lisa P. FOC 11:10

Wesdemiotis, Chrys ThPA 013, FOA 10:10, ThPA 014, ThPA 006, TPA 022, TPA 002

West, Andrew MPF 286

Wester, Dennis W. WPF 160

Westerduin, Pieter ThPE 175

Westmacott, G. R. ThPF 192

Westman, Ann ThPF 208

Westmore, J. B. ThOF 11:10

Westmore, John MPD 193, MPD 197

Wexler, Anthony WPI 287, WPI 284

Whalen, Kevin I-1 ThPB 051

Wheelan, Pat TPE 173

Wheeler, C. TPG 254

Wheeler, K. P. TPG 291

Wheeler, Kevin TPB 064, WPG 178, TPG 290, TPC 117

Wheeler, Patrick D. WOA 10:50

White, Dineen J. WPG 183

White, Earl ThPG 291, ThPG 293

White, Forest M. WPA 018, ThPD 133, ThPB 065, ThPF 202

White, John MPD 163

White, Mark WPD 088

White, Robert WPG 173

White, Robert J. MPF 245, FOC 11:10

Whitehill, Andy ThPG 284

Whitehill, Andy B. ThPG 287

Whitehouse, Craig MPB 055, WPH 205, ThOD 11:50

Whitehouse, Craig M. WPH 218, MPB 057, TPB 083, MPB 059

Whitehouse, Larry TPE 163

Whitesides, George M. WPH 232

Whiting, Andrew TPA 007

Whitman, Christian P. MPE 225

Whitney, Jeffrey MPC 107

Whitney, Jeffrey L. MPC 108, TPE 202, TPB 061, TPC 119

Whittal, Randy M. WPI 245, MOC 11:50, ThPF 209, ThPF 212

Whitton, Margie MPF 291

Wiberg, Karin MPC 118

Wickham, Juanita WPI 263

Widger, William R. TPE 152

Widlanski, Theodore S. ThPD 135

Widmer, H. Michael ThPE 181

Wiedmann, Fred A. ThPA 013

Wiedmann, Fred ThPA 014

Wight, Helen WPB 050

Wilcox, M. D. MPF 287

Wilhelm, Jeffrey A. WPG 171, MPF 245

Wilhelm, Ulf ThPB 055

Wilkerson, Charles W. MPD 144

Wilkes, Jon G. MPB 074, MPD 170, MPD 169, TPB 095

Wilkins, Charles L. TPB 108, TPB 090, TPA 043, ThPF 203

Wilkins, Pat ThPG 276

Wilkinson, William R. WOA 04:40
Willett, Gary WPI 295

Williams, David E. TPD 142

Williams, David T. WPF 135

Williams, Evan TOC 04:00

Williams, Evan R. TOC 11:50, WPH 224, MPA 043

Williams, John B. WPI 274

Williams, Jon ThPE 167

Williams, Peter ThOB 05:00, WPI 269, WPI 268, WPG 195, WPI 252

Williams, Ted L. WOE 10:30

Williams, Todd D. ThPA 011, TOA 10:30

Williams, Tracie L. TOF 10:30

Williamson, Brian MPA 025

Williamson, D. H. MPB 068

Willoughby, Ross WOB 04:40, TPC 124, WPH 215, WPH 216

Willoughby, Ross C. TPB 094

Wilm, M. S. TPF 231

Wilm, Matthias ThPG 270, MOD 09:30, ThOC 11:30, FOB 09:30, WPB 046, TPG 276

Wilner, Keith TPE 197

Wilson, Ian D. TPE 168, TPE 191

Wilson, Ray WPB 045, WPB 044, ThPB 078

Wilson, Terry MPC 136

Wilton, Amanda N. TPE 180

Winans, Randall E. ThOE 10:30, WPC 069

Winders, Andrew TPA 011

Wing, David R. ThPE 152

Winger, Brian TPB 078, TPA 041

Winnik, Witold TPA 031, ThPC 123

Winslow, L. D. WPG 181, WOA 12:10

Winters, Conrad MPC 138

Wintner, Edward A. TPC 123

Wise, Marc B. WPB 041

Wise, Marcus WPF 133, WPF 146

Wishnok, J. S. TPD 129

Witkowska, H. Eva ThPG 295

Wold, Chad WPA 006

Wolf, Susan TPG 265

Wolfe, Henry MOD 11:10

Wolfe, Tracy L. MPC 123

Wolfender, Jean Luc TPE 182

Wollnik, Hermann MPB 079, MPB 087, MPB 098

Wolter, M. A. TPF 231, TPF 239

WOLUCKA, B. A. ThPE 182

Wong, Carla WPF 147

Wong, Diane MPF 242

Wong, Jennifer MPD 177

Wong, Mel MPE 214

Wong, P. MPC 112

Wong, R. Y. TOB. 11:30

Wood, Troy D. TPG 289, MPA 041, MOC 04:20

Woods, A. S. WPI 237, ThPG 280

Woods, Amina MPF 240, ThOB 11:10

Woods, James MPF 253

Woolen, Stan W. FOC 10:10

Worrall, Timothy A. TPF 216

Worthy, Suzanne WPF 131

Wright, Adrian TPB 062 
Wright, L. James ThPA 044

Wright, Scott WPI 279, WPI 280

Wronka, John MPB 083, MOC 12:10, ThPF 193

Wu, Henry Y. WPG 177, WPG 168, WPG 176

Wu, Jiang WPI 254

Wu, Jiangyue ThPF 199, ThPE 180, ThPB 061, WOC 11:50

Wu, Jingtao TPB 089

Wu, Kuang Jen ThOD 11:30, WPI 264, WPI 263

Wu, Kuen Yuh TPD 126

Wu, Qinyuan TOC 10:30, ThPB 066, TPA 001

Wu, Wu-Nan TPE 159, TPE 160

Wuerker, Ralph ThPC 099

Wulser, Kurt W. MPB 096

Wunderlin, Markus TPG 256

Wysocki, Vicki TPG 266, ThPA 028, TPA 015, TPA

045, TPA 030, TPA 044, FOE 10:10

Wyttenbach, T. TPB 076

Xia, Ming MPB 056

Xiang, Xinzhen Jane MPC 137

Xiao, Guang TPE 198

Xie, Xiaoling FOD 12:10

Xin, Tianpei WPA 018

Xiong, Yansan ThPF 223, WOD O4:00

$\mathrm{Xu}$, Allan WPG 184

$\mathrm{Xu}$, Linxiao TPF 224

$X u$, Ming WPF 129

$X u$, Naxing TPE 210

$\mathrm{Xu}$, Xiaoming MPE 223

$X u$, Yaodong MPE 234

Xu, Young WPA 014, WPA 013, WPA 017

Xue, Weiling MPD 178

Xuewu, Jiang ThOC 05:00

Yablokov, Michail ThPB 053

Yakovlev, Boris S. MPB 065

Yalcin, Talat TPA 009

Yamada, Hitoshi MPA 049

Yamakawa, Yoshio TPG 262, MPF 275

Yamamoto, Ralph MPF 263

Yan, Lin MPF 244

Yang, George TPB 081

Yang, Hou-Jun ThPG 253, WPI 238, ThPF 230

Yang, Lily WPF 154

Yang, Mo MPC 115

Yang, Sheng ThPA 010

Yang, Y. ThPE 156

Yang, Yang ThPD 144

Yang, Yarjing ThPA 009, TPA 014

Yao, Flora ThPE 173

Yao, Jie ThPF 203, TPA 043

Yates, Hilary WPC 063, WPC 062, WPC 066, MOC 10:10

Yates, John MPF 294, TPG 279

Yates, John R. ThOC 04:00, TPG 278

Yates, John R. III ThOB 12:10, ThPG 283
Yates, Nathan A. TPG 264

Yeager, Mark WPH 207

Yeh, Che-Hung ThPG 266

Yeh, Chen-Sheng WPA 017

Yen, Ten-Yang MPE 216, TPD 127

Yeola, Suresh N. WPG 199

Yergey, Alfred L. MPE 234

Yergey, James TPE 167, ThPD 134

Yeung, Bernice MPF 269

Yhard, G. Bradley WPD 093

Ying, Z. Charles ThPA 001

Yinon, Jehuda TOB 04:00

Yip, Tai-Tung ThPF 242, ThPF 243

Yongwu, Liang TPB 079

Yoo, Jong Shin MPC 132

Yoo, Ryong TOF 05:00

Yoshida, Keiichi ThPE 171

Yoshidome, K. WPB 039

Yost, Richard ThOC 10:30, WPI 270, ThPD 140, TPE 171

Yost, Richard A. ThOE 09:30, ThPC 101, ThPC 100, TPA 032, TOF 10:30, TPA 035, ThOE 10:10, ThPC 115

You, Jianzhang TPB 094

Young, J. P. WPD 086

Young, Larry WPG 196

Youngquist, R. Scott TPC 114

Yousef, Ibrahim TPE 179

Yu, Chungping ThPD 135

Yu, Jim MPF 253

Yu, Qitao MPE 215, MPF 273

Yu, Wen WOD 11:10

Yu, Zhonghua MPF 290

Yuan-Ping, Yang TPE 204

Yuji, Reiko MPF 257, MPF 256

Zacchello, F. WPG 201

Zagorevski, Dmitri FOA 12:10, ThOA 05:00

Zaia, Joseph TPD 145

Zaikin, Vladimir MPC 100

Zamborelli, Tom ThPE 172, ThPG 268

Zamecnik, Jiri WPG 180

Zefirov, Nikolay MPC 131

Zell, Manfred TPE 172, TOA 04:40

Zembrowski, William TPE 175

Zeng, Chenhui ThPE 149

Zeng, Jianru ThPF 191, ThPE 169, WPI 247

Zenobi, Renato MPD 143, WPI 281

Zhan, Qaio WPI 281, MPD 143

Zhang, Wenzhu TPG 281

Zhang, Haiying WPI 251

Zhang, J. WPH 229

Zhang, Jian WPI 242

Zhang, Jie ThPD 144

Zhang, R. ThOA 10:10

Zhang, Tian-lan MPA 047

Zhang, Tianlan MPA 028

Zhang, X. Kate ThPF 205, TPA 016

Zhang, Xin MPE 234

Zhang, Yu TPG 292 
Zhang, Z. TPG 285

Zhang, Zhongqi FOD 11:30

Zhao, Jamie WPG 192, WPG 176, WPG 168

Zhao, Sean WPB 034

Zhao, Xinjin WPC 067

Zhao, Yingming ThOB 04:40

Zhao, Zhiyang TPD 139

Zhao, Zhongxi TPB 077, TPB 087

Zhao, Zhongxi (Zack) TPB 085, TPF 228

Zheng, Kefei FOB 11:30

Zhong, Wenquing TPA 045

Zhou, Feimeng WOB 11:10, WPH 221, MOF 10:30, WPH 222

Zhou, Jie ThPF 217, ThPF 218
Zhou, Joe ThPC 097, MPC 120, ThPC 098, ThOC 11:50, ThPC 084, ThPC 095, ThPC 094

Zhou, Ping WPI 266

Zhu, Y. F. MPF 237

Zhu, Ying ThPD 132

Ziegler, J. WPE 123, WPE 124, WPE 122, WPI 290, WPA 002

Zilg, Harald A. MPF 282

Zlotkin, Eliahu ThPG 272

Znamirovschi, Calin G. WPD 092

Zoller, David WPC 070

Zubarev, Roman ThPA 035

Zuckerman, Ronald N. TPC 117

Zyk, Nikolay MPC 131 